\title{
Analysis of ring operation from south Andaman Fish Landing Centres (FLC) ${ }^{*}$ M. Kaliyamoorthy ${ }^{1}$, S. Dam Roy ${ }^{2}$ and V.K. Sahu ${ }^{3}$
}

\author{
${ }^{1}$ PRIST University, Thanjavur \\ ${ }^{2}$ Central Island Agricultural Research Institute, Port Blair, \\ ${ }^{3}$ Department of Zoology, JNRM, Port Blair \\ *Corresponding Author \\ Email: kamuthy@gmail.com
}

Received : 25.02.2020; Accepted : 22.04.2020

\begin{abstract}
A study was carried out during 2014-2018 to find out the major fish catches through the Gear Ring net at the coast of Andaman Islands. The data were collected from four major Fish Landing Centres (FLC) viz Junglighat, Dugnabad, Guptapara and Wandoor which are located at South Andaman. Altogether 1097 FLC visits have been carried out during the study period at all four FLC with an average of 219 visits / year. Altogether 1379 operations of Ring net were observed from two FLC i.e. Junglighat (1322) and Dugnabad (57) amongst four FLC. The total fishes captured were 1609 tons from the two FLCs, i.e. 1594 tons from Junglighat FLC and 15 tons from Dugnabad FLC respectively. The contribution of gear the Ring net was 41.5 $\%$ amongst the other gears operated from all four FLCs. The major fish catch by ring net were Scombrids (52.3\%), followed by Sardines (22.4\%), Carangids (21.3\%), Anchovies (2.7\%) and others (1.2\%). 39 ring net fishing ground identified during the period around the coast of Andaman Islands. The maximum fish catch occurred at around the coast of Havelock Island (15.5\%) followed by Rutland (10.5\%), Chidiatappu (8\%), Shoal Bay (7.6\%), Baratang (7\%), Carbyns cove (6 \%), Burmanallah (5.2\%), Madhuban (5.2\%), Out-drum Island (4.7\%), Mayabunder (4\%), Colinpur (3.3\%), Long Island (3.3\%), Wandoor (2.1\%) etc and minimum fish catch was observed at the coast of Pachim Sagar $(0.02 \%)$.

PFZ forecast disseminated to the Ring netters and validated during the study period. Forty nine operations of gear the ring net were observed at PFZ with an average of 9.8 operations /year. The fishes captured from the PFZ were 91.1 tons with an average of $18.2 \pm 5.6$ tons / year. Similarly 49 operations of ring net were also observed at Non-PFZ with an average of 9.8 operations /year. The fishes captured from the Non PFZ were 23.77 tons with an average of $4.5 \pm 1.5$ tons / year.

The Length-weight measurements of the pelagic fishes viz Atule mate, Decapterus russelli, Sardinella albella, Sardinella sirm, Herklotsichthys quadrimaculatus and Rastrelliger kanagurta caught from both the zones have been done. 12 Class Intervals with respect to length were observed from the catch of all the fishes i.e. 81-100 mm, 101-120, 141-160 mm,.......301$320 \mathrm{~mm}$. In the Class Intervals, the length category 121-140 mm was the most representating class for Atule mate with 128 specimens (19.1\%) at PFZ and 159 specimens (23.7\%) at Non-PFZ respectively. It has been observed for other fishes i.e. for Decapterus russelli, Sardinella albella, Sardinella sirm, Herklotsichthys quadrimaculatus and Rastrelliger kanagurta which collected from the PFZ were class 161-180 mm with 172 specimens (48.6\%), 141-160 mm with 195 specimens (53.1\%), 221$240 \mathrm{~mm}$ with 177 specimens (48.2\%), 121-140 mm with 189 specimens (47.7\%) and 261-280 with 199 specimens (30.9\%) respectively. The same species were collected at the Non-PFZ were 141-160 with 173 specimen (49.0\%), 121-140 with 194 specimens (53.3\%), 221-240 with 159 (45.4\%), 101-120 with 173 (49.4\%) and 201-220 with 144 specimens (23\%) respectively.

The water samples have been collected during day time from various sites of PFZ and Non-PFZ during the study period and analysed. The average visibility at PFZ and Non-PFZ were $15.49 \pm 0.28 \mathrm{~m}$ and $16.74 \pm 0.25$ respectively. The average dissolved oxygen (DO) at PFZ and Non-PFZ were $6.30 \pm 0.05 \mathrm{mg} / \mathrm{lit}$ and $5.94 \pm 0.06 \mathrm{mg} / \mathrm{lit}$ respectively. Due to density of phytoplankton and Eddies the dissolved oxygen increased at PFZ than Non-PFZ. Alkalinity was at PFZ $111.16 \pm 1.09 \mathrm{ml} / \mathrm{lit}$ and Non PFZ $113.68 \pm 1.28 \mathrm{ml} /$ lit respectively.
\end{abstract}

Figures : 09

References : 40

Tables : 07

KEY WORDS : Andaman, Dugnabad, Guptapara, Fish landing centre, Junglighat, Ring net, Wandoor.

\section{Introduction}

Fisheries play an important role in Indian economy and it is one of the most important economic activities along the coastal areas ${ }^{11}$. According to $\mathrm{FAO}^{12}$ total capture fisheries production of India was estimated about
93 million tons ${ }^{38}$ and the contribution from marine capture fisheries was about $90 \%$. Annual marine fishery potential of Indian EEZ is estimated as 3.93 million tons and the sector - wise contribution of marine fish landings are $71 \%$ in mechanized sector, $24 \%$ in motorized sector and $5 \%$ in artisanal sector? 

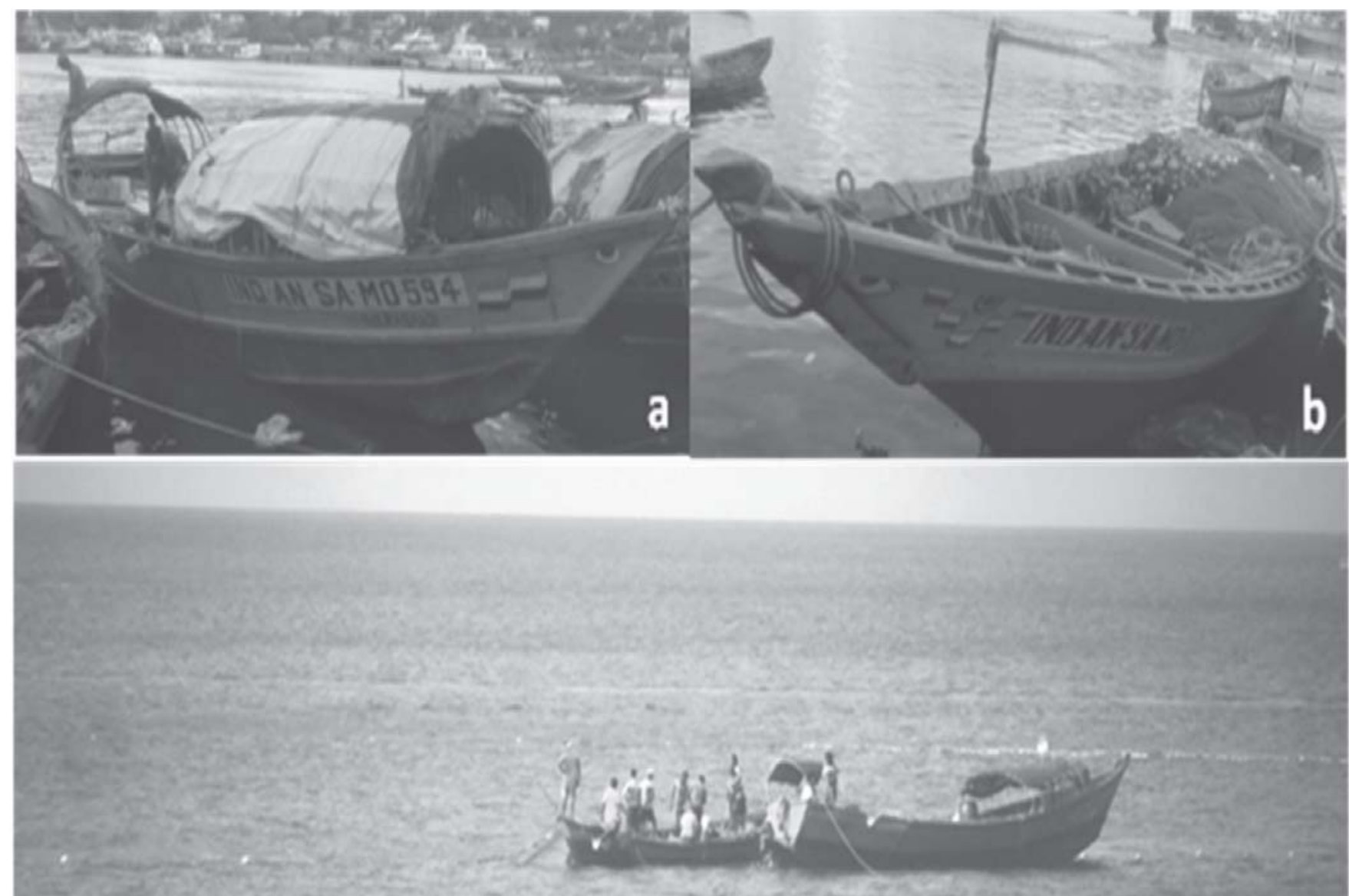

Fig.1: Dinghies for Ring net operation at Andaman Islands (a) Main dinghy, (b) Net dragging dinghy and (c) Occurring of Ring net operation at North Bay, South Andaman.

The Andaman Sea is situated in the southern part of the Bay of Bengal between $92^{\circ} 12^{\prime} \mathrm{E}$ and $93^{\circ} 57^{\prime} \mathrm{E}$ longitude and between $6^{\circ} 45^{\prime} \mathrm{N}$ and $13^{\circ} 41^{\prime} \mathrm{N}$ latitude with $10^{\circ} \mathrm{N}$ channel separating Andaman group and Nicobar group of islands. The total length of these islands is about $700 \mathrm{~km}$ covering an area of about 8249 sq. $\mathrm{km}$ with a total coastline of $1,192 \mathrm{~km}$. These islands are generally oceanic in nature and comprise an Exclusive Economic Zone (EEZ) of 0.6 million $\mathrm{km}^{2}$, i.e. about $28 \%$ of total EEZ of the country. The continental shelf is limited with an estimated area of $16,000 \mathrm{~km}^{2}$ and the sea is very deep within few $\mathrm{km}$ from the shore ${ }^{2}$. The Fishery Survey of India estimates that these islands are home to $9.2 \%$ demersal, $57.1 \%$ coastal, and $33.7 \%$ oceanic fish stocks ${ }^{3}$.

Recognition of Potential Fishing Zones (PFZ) occupies an understanding of oceanic processes and interaction of hydro-biological parameters ${ }^{9}$. Many of pelagic fish species are known to concentrate at present boundaries especially in areas with sharp horizontal temperature gradients. Indirect methods of monitoring selected parameters such as SST and Chlorophyll-a (phytoplankton pigments) at surface of the sea from satellites are found to be very ideal, as it supplies high repetivity and large special coverage (INCOIS).

The purse seine belongs to the surrounding net class in which fish are surrounded not only from the side but also from below, letting them to be caught in deep waters ${ }^{13}$. Purse seine is named from the feature that along the bottom of the net are a number of rings, a rope passes through the rings, and when pulled the bottom of the seine is closed, like a purse, preventing the fish from escaping. The purse seine is a preferred technique for capturing schooling fish species, close to the surface such as sardines, mackerel and anchovies ${ }^{30}$.

\section{Review of the Literature}

The total fishery resources of Andaman sea is about 1.48 lakh tonnes of which 56,000 tonnes are pelagic, 32,000 tonnes are demersal and 60,000 tonnes are 
CARANGIDS

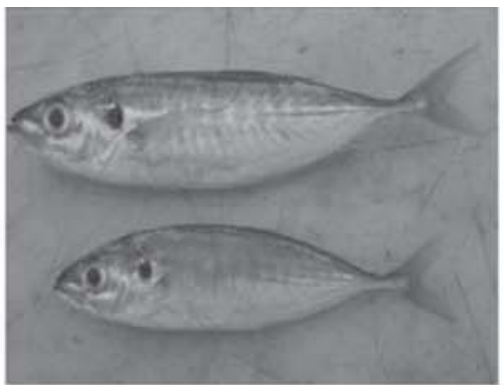

Atule mate

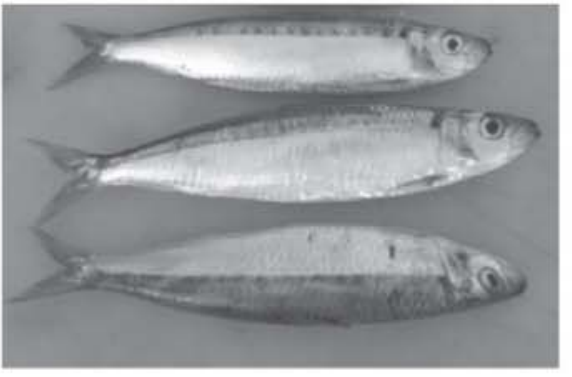

Decapterus sp.

SARDINES

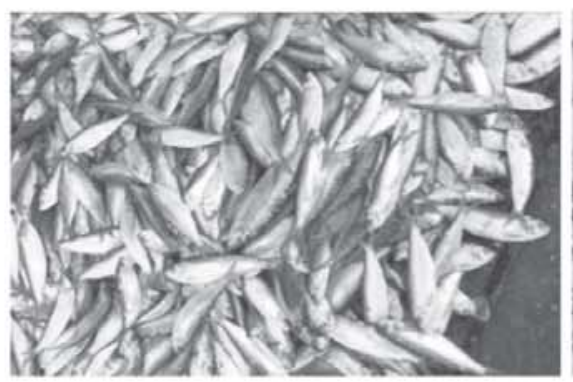

Sardinella species

SCOMBRIDS

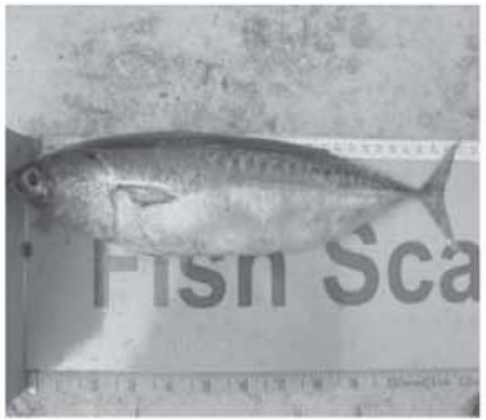

Little tuna (Auxis sp.)

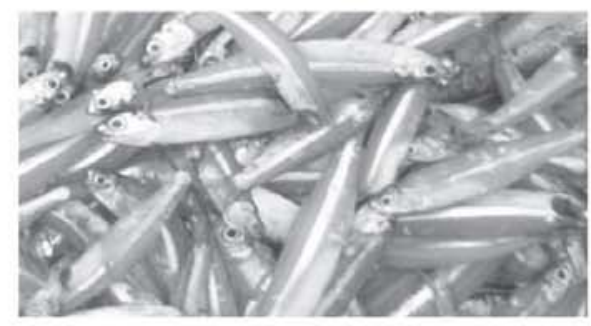

Anchovy (stolephorus sp.)

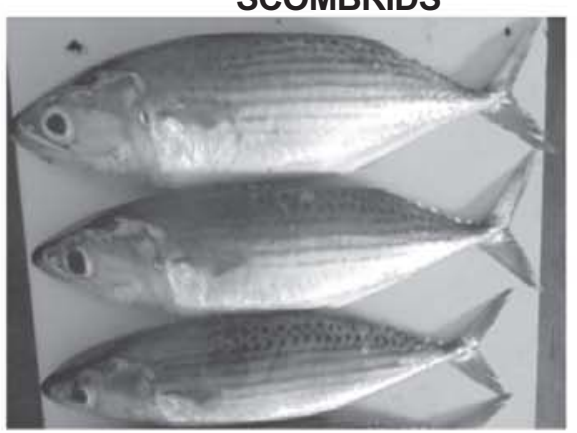

Mackerel (Rastrelliger sp.) OTHER FISHES

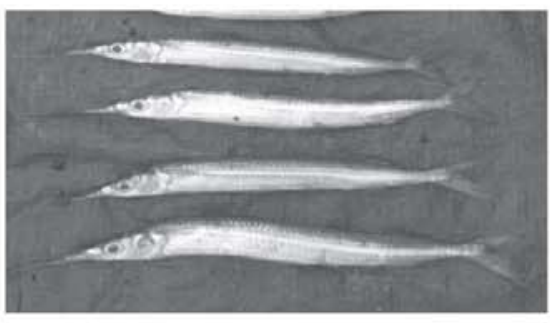

Half beak (Hemiramphus sp.)

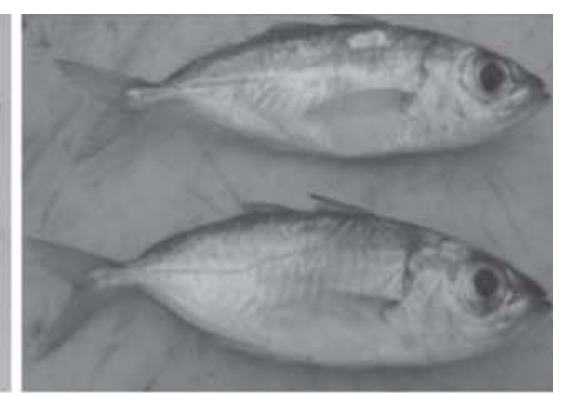

Selar sp.
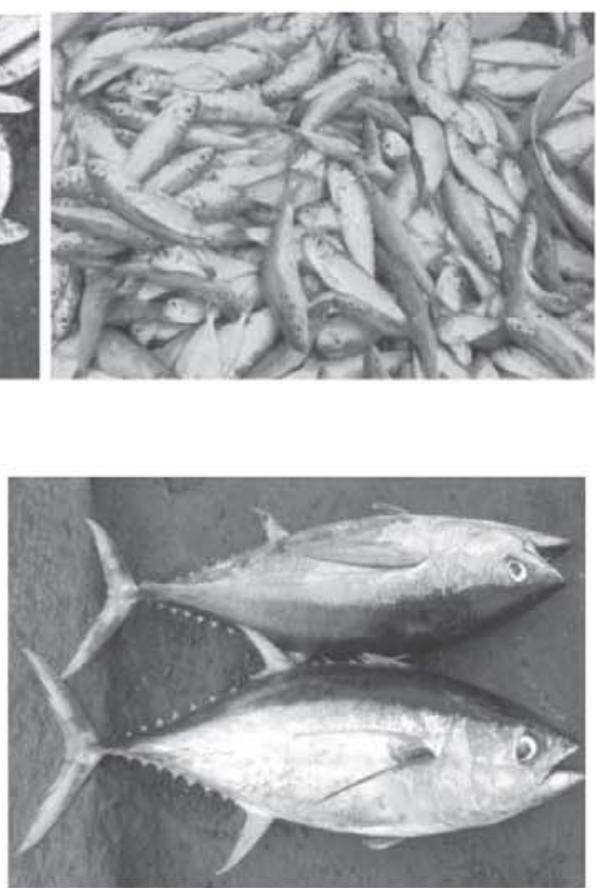

Yellow fin tuna (Thunnus sp.)

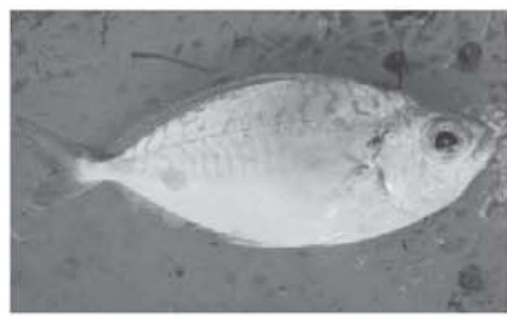

Silver belly (Lieognothus sp.)
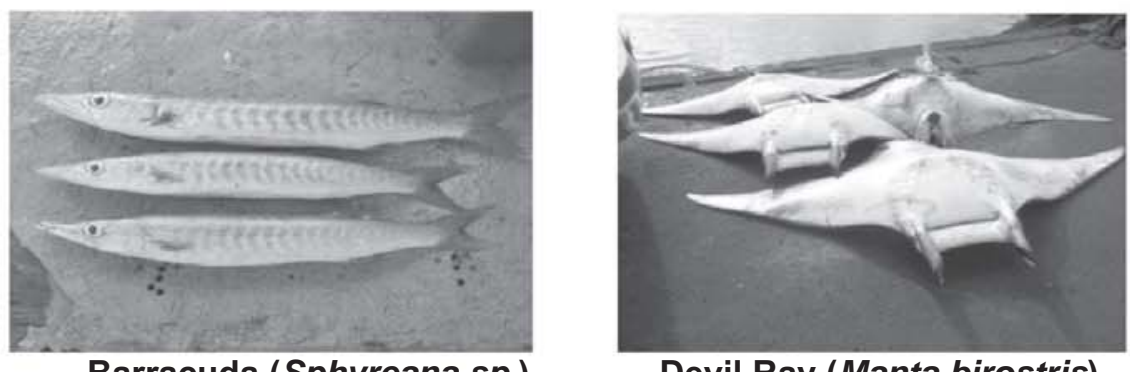

Barracuda (Sphyreana sp.)

Devil Ray (Manta birostris),

Fig. 2. Some Pelagic Fishes 
TABLE-1: FLC visits and total fish catch at Junglighat, Dugnabad, Guptapara and Wandoor

\begin{tabular}{l|l|c|c|c|c|c|c}
\hline FLC & Year & $\mathbf{2 0 1 4}$ & $\mathbf{2 0 1 5}$ & $\mathbf{2 0 1 6}$ & $\mathbf{2 0 1 7}$ & $\mathbf{2 0 1 8}$ & Total \\
\hline Junglighat & Total visits (N) & 177 & 129 & 137 & 136 & 113 & 692 \\
\hline & Total catch (ton) & 914.1 & 665.7 & 747.2 & 802.3 & 696.5 & 3825.9 \\
\hline Dugnabad & Total visits (N) & 24 & 21 & 54 & 42 & 28 & 169 \\
\hline & Total catch (ton) & 5.0 & 5.3 & 7.0 & 7.7 & 5.4 & 30.4 \\
\hline Guptapara & Total visits (N) & 27 & 18 & 35 & 28 & 21 & 129 \\
\hline Wandoor & Total catch (ton) & 2.5 & 1.9 & 2.8 & 2.7 & 2.5 & 12.4 \\
\hline & Total visits (N) & 10 & 15 & 35 & 28 & 19 & 107 \\
\hline
\end{tabular}

oceanic. The marine fishery in the ANI is dominated by pelagic catches, which comprise about $60 \%$ of the total catch. Gillnet (27\%), hand-line (54\%), long-line (5\%), and ring net/seine (1\%) are the major types of fishing gears used in the Andaman fishery ${ }^{14}$. The exploitation of fishery resources at present is restricted to coastal waters ${ }^{29}$. There is no organized offshore fishing from Andaman base fishery ${ }^{8}$.

Although $\mathrm{ANI}$ is unique in possessing high quantity of harvestable fishery resources, current marine capture fisheries remain greatly underutilized. The marine fish production in the Islands amounted to 30,000 tonnes constituting a meagre $19 \%$ of the estimated potential ${ }^{8}$. The ring seine is targeted at shoaling pelagic fishes like scombrids, carangids, clupeids and anchovies ${ }^{20}$. Indian Remote Sensing Satellite P4 Ocean Colour Monitor (IRS P4 OCM) derived chlorophyll concentration and National Oceanographic Aerospace Administration Advanced Very High Resolution Radiometer (NOAAAVHRR) derived Sea Surface Temperature (SST) images have been used to characterise the relationship between biological and physical variables in coastal waters and it was observed that both chlorophyll concentration and SST were inversely correlated ${ }^{37}$.

Mesoscale eddies increase biological productivity by vertical and horizontal mixing of the water column in the pelagic zone ${ }^{40}$. The eddies reduce thermocline depth and bring nutrients to the photic zone, improving the productivity in stratified tropical and subtropical regions of the oceans ${ }^{21}$. Eddies increase the local productivity in the oligotrophic regions of tropical oceans ${ }^{18}$. Mesoscale eddies influence productivity at every trophic level, such as the primary production ${ }^{4,22,32}$ and concentration of zooplankton, micro-nekton ${ }^{31}$ and plankton feeders ${ }^{27}$, which in turn form a forage base and attract tertiary-level producers (tunas, marlin, turtles, sea birds, and cetaceans). IRS P4 OCM were disseminated through different modes viz., Digital display boards, e-mail, telephoning/text messaging, radio, community networking and distribution of print-outs in person to the targeted fishermen and the efficacy was tested ${ }^{16}$.

Although Andaman and Nicobar Islands (ANI) are unique in possessing high magnitude of harvestable fishery resources ${ }^{8}$ a significant increase (30\%) in total catch by following PFZ forecasts has been documented from Andaman and Nicobar Islands ${ }^{15}$. Some workers ${ }^{17}$ have documented the species composition and food and feeding behaviours of fishes harvested within and outside PFZs. However, these Islands (ANI) are having cloud covers for about eight months of the year, which obscures optical and thermal imagery and hinders the generation of PFZ advisories ${ }^{16}$.

\section{Materials and Methods}

A study was carried out to investigate the major fish catches through the Gear Ring net consecutive years from 2017-2018 at four fish landing centres viz Junglighat, Dugnabad, Guptapara and Wandoor in South Andaman. Every fish landing center was visited periodically according to the landings of fishes to get the proper landings data. 
TABLE.2: Fish Landings through different gear operation at South Andaman

\begin{tabular}{|c|c|c|c|c|c|c|c|}
\hline Year & 2014 & 2015 & 2016 & 2017 & 2018 & Total & Ave with SE \\
\hline $\begin{array}{l}\text { Number of all gear } \\
\text { operations }\end{array}$ & 1551 & 1252 & 1296 & 1279 & 1367 & 6745.0 & $1349.0 \pm 54.0$ \\
\hline Total catch (in ton) & 923.7 & 675.1 & 759.1 & 815.1 & 707.7 & 3880.6 & $776.1 \pm 43.8$ \\
\hline $\begin{array}{l}\text { Number of ring net } \\
\text { operations }\end{array}$ & 298 & 277 & 297 & 253 & 254 & 1379 & $275.8 \pm 9.6$ \\
\hline $\begin{array}{l}\text { Total catch through } \\
\text { Ring net (in ton) }\end{array}$ & 398.1 & 314.9 & 324.6 & 308.4 & 263.3 & 1609.2 & $321.8 \pm 21.8$ \\
\hline Scombrids (in ton) & 209.4 & 124.2 & 178.2 & 175.1 & 155.4 & 842.3 & $168.5 \pm 14.0$ \\
\hline Carangids (in ton) & 106.6 & 77.0 & 52.7 & 57.3 & 48.9 & 342.6 & $68.5 \pm 10.7$ \\
\hline Sardines (in ton) & 79.84 & 97.52 & 80.30 & 55.6 & 47.4 & 360.7 & $72.1 \pm 9.1$ \\
\hline Anchovies (in ton) & 0.35 & 11.48 & 10.30 & 13.4 & 8.5 & 44.0 & $8.8 \pm 2.3$ \\
\hline Other fishes (in ton) & 1.9 & 4.7 & 3.1 & 7.0 & 3.1 & 19.6 & 3. $9 \pm 0.9$ \\
\hline
\end{tabular}

The Ring net is the main gear used to capture the pelagic fishes like Scombrids included Indian Mackerels - Rastrelliger sp., rarely Little tuna - Auxis thazard, Yellow fin tuna -Thunnus albacares, Skipjack tuna - Katsuwonus pelamis etc., Sardines included all the Sardinella species, small Carangids and Anchovies in large quantity (i.e. 0.5 to 5 ton/ boat) from Andaman Islands. The other fishes like Milk fish (Chanos chanos), Silver bellies (Lieognothus sp.,), Silver buddies (Gerres sp.,), Barracuda (Sphyreana sp.), Devil Ray (Manta birostris), Reef sharks etc., were also observed in catch occasionally. Schools of sardines, mackerel, skipjack and other varieties of fishes have been very frequently sighted around the islands, which provide much scope for purse seine operations and also comprised as highly economic varieties.

Ring net was twine 1.5/2, mesh size $18 \mathrm{~mm}$, with mesh length $1000 \mathrm{~m}$ and depth $50 \mathrm{~m}$. Two types of wooden dinghies which were used in each fishing operation for ring net i.e. main dinghy with the length 13 to $17 \mathrm{~m}$, width 2 to $3 \mathrm{~m}$ and height 1.5 to $2 \mathrm{~m}$ having inboard diesel engine with 10 to $20 \mathrm{hp}$ for transporting and storing the capturing fishes and net handling dinghy with net rotating engine engaging in the fishing operation (Fig. 1). The word for small boats in these islands is dungi used as a tender for larger vessels ${ }^{5}$. All traditional craft are locally made with the wood of 'taung-pienne' (Artocarpus chaplasha) as the preferred choice. These craft are paddled, punte, or sailed in waters upto $20 \mathrm{~m}$ deep across the length of the Andaman Islands ${ }^{24}$. The commercial fishery of the Andaman Islands has grown steadily from its modest start of a few plank built canoes to a fleet of 2,813 craft. More than a half of these $(1,465)$ are still non-motorised ${ }^{1}$.

The maximum Ring net operations, occurring from Junglighat FLC is maintained by the Department of Fisheries, Andaman and Nicobar Administration; which is extending loading and unloading facilities with proper arrangement of security. The fishing harbours, fish landing centres (FLCs) are significantly important meeting places for artisanal fishers, buyers, traders, government officials and those providing services to a fishing community ${ }^{33}$.

The Potential Fishing Zone (PFZ) forecasts by the Indian National Centre for Ocean Information Services (INCOIS) have been proved beneficial to the fish catcher of the Islands ${ }^{15,17}$. PFZ forecasts have been disseminated to the targeted fishermen of four landing centres during the visits. Gear wise fish landings were recorded in different gears like ring net, various type of gill net, Anchor net, Trawl net, Hand liners and Long liners. Identical fishing vessels of different types have been used for carrying out simultaneous validation experiments in PFZ 


\begin{tabular}{|c|c|c|c|c|c|c|c|c|c|c|c|c|c|c|c|c|c|}
\hline \multicolumn{2}{|r|}{ 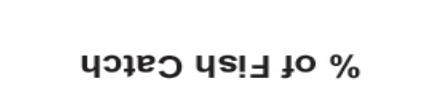 } & $\stackrel{\nwarrow}{\sim}$ & ষ্ণ & $\underset{\dot{\sigma}}{\tilde{\sigma}}$ & 응 & $\stackrel{\sim}{\infty}$ & $\frac{N}{\sigma 0}$ & $\begin{array}{l}\mathscr{8} \\
10 \\
i\end{array}$ & $\begin{array}{l}\text { Oొ } \\
\infty \\
\infty\end{array}$ & $\frac{⿱ 亠}{0}$ & $\begin{array}{l}\text { ठ } \\
\text { ○ }\end{array}$ & $\stackrel{\text { m }}{\text { ले }}$ & $\begin{array}{l}\text { 员 } \\
0\end{array}$ & $\stackrel{\mathscr{S}}{\rightleftharpoons}$ & $\frac{M}{0}$ & 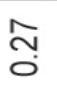 & $\begin{array}{l}\text { ஜึ } \\
\text { مٌ }\end{array}$ \\
\hline \multirow{3}{*}{ 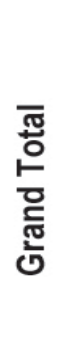 } & 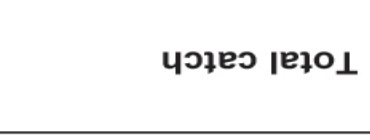 & $\begin{array}{l}\text { \& } \\
\text { ণ্ }\end{array}$ & 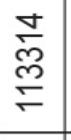 & $\begin{array}{l}\stackrel{R}{8} \\
\mathbb{8}\end{array}$ & 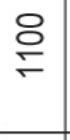 & $\begin{array}{l}\text { 유 } \\
\text { D্ }\end{array}$ & $\begin{array}{l}\stackrel{0}{\mathbb{N}} \\
\underset{\infty}{\infty}\end{array}$ & 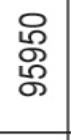 & 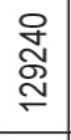 & ్ㅗ & 오 & 유 & চి & 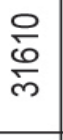 & $\begin{array}{l}\stackrel{8}{\circ} \\
\frac{\infty}{\leftarrow}\end{array}$ & ষ্ণি & $\begin{array}{l}\text { ণ } \\
\text { ণ } \\
\text { }\end{array}$ \\
\hline & †әu yo uo!̣esədo & $\mathscr{q}$ & $\stackrel{\widehat{o}}{\text { D }}$ & 으 & 0 & గొ & $\stackrel{\mathscr{m}}{\leftarrow}$ & กิ & 芯 & $\sim$ & $m$ & శా & 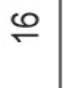 & $\mathscr{\wp}$ & $\cong$ & の & ণoo \\
\hline & $d !$ & N & $\stackrel{\sim}{\simeq}$ & $\bullet$ & $n$ & হ & ஜ & $\varphi$ & $\stackrel{\aleph}{r}$ & $\bullet$ & $\leftarrow$ & 오 & $\sigma$ & ஜ & $\stackrel{m}{\square}$ & $\bullet$ & $\stackrel{\sim}{N}$ \\
\hline \multirow{3}{*}{ 유 } & 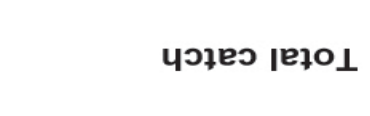 & 员 & 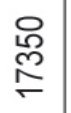 & ণ্ণ్ & 유 & ర్రి & $\begin{array}{l}\circ \\
\stackrel{0}{0} \\
\stackrel{0}{\Gamma}\end{array}$ & $\frac{8}{\stackrel{8}{E}}$ & $\begin{array}{l}\text { 음 } \\
\text { o } \\
\stackrel{\rho}{\longrightarrow}\end{array}$ & 옹 & o & $\stackrel{\circ}{\stackrel{\circ}{\rightleftarrows}}$ & ণ্సি & 웜 & ৪্ল & 음 & 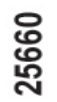 \\
\hline & 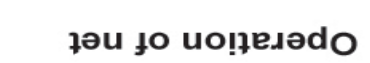 & $\stackrel{m}{\sim}$ & 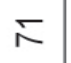 & $\sim$ & $m$ & $\stackrel{2}{\leftarrow}$ & $\ddot{m}$ & প్ల & テ & $\sim$ & ○ & ๗ & 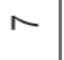 & 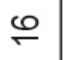 & $\infty$ & $\bullet$ & $\Re$ \\
\hline & d! & $\infty$ & প্ল & $\sim$ & $\sim$ & $\sigma$ & $\approx$ & 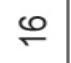 & $\check{\sim}$ & $\sim$ & 0 & $\approx$ & $\nabla$ & o & م & $m$ & ్ల \\
\hline \multirow{3}{*}{$\bar{\sigma}$} & чग‡е & 용 & 을 & 은 & ర్లి & ర్రి & $\begin{array}{l}\text { 怘 } \\
\stackrel{0}{\sim} \\
\stackrel{2}{\sim}\end{array}$ & $\frac{8}{\bar{N}}$ & $\begin{array}{l}\text { ঃ্ల } \\
\stackrel{m}{\varpi}\end{array}$ & 웅 & 0 & 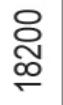 & ণ্ণি & প্ণি & ষ্ণ & 8 & $\underset{\text { స్లా }}{\text { No }}$ \\
\hline & łəu jo uo!̣esədo & $\cong$ & 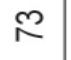 & $\sim$ & $\sim$ & $\cong$ & $\hat{\sim}$ & $\stackrel{\infty}{\infty}$ & $\mathscr{N}$ & $\checkmark$ & o & న & $\nabla$ & 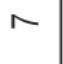 & $\infty$ & $\sim$ & $\stackrel{\bullet}{\circ}$ \\
\hline & d! $\perp$ & 6 & $\bar{m}$ & $\sim$ & $\sim$ & N & $\stackrel{\varphi}{\check{1}}$ & $\stackrel{\infty}{\infty}$ & 巳 & $m$ & 0 & $\approx$ & $m$ & $\nabla$ & $m$ & $\sim$ & ా্ల \\
\hline \multirow{3}{*}{ 으 } & чગ્Е & గ్లి & 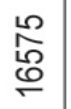 & 음 & ి్లి & ৪ి & 윰 & 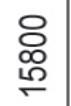 & 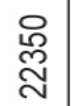 & ర్లి & 오 & 옴 & 오 & 읅 & 옹 & రి & శ్ర్గి \\
\hline & †əu fo uo!̣esədo & $\sim$ & $\mathscr{\checkmark}$ & $m$ & - & 0 & $\bar{\sim}$ & $\check{\sim}$ & $\bar{m}$ & $\leftarrow$ & $m$ & $\infty$ & $\sim$ & $\bar{\sim}$ & - & - & $\stackrel{m}{\leftarrow}$ \\
\hline & d! $\perp$ & $\leftarrow$ & 요 & - & - & $\leftarrow$ & a & $\stackrel{m}{\rightleftharpoons}$ & $\mathscr{N}$ & - & - & $\nabla$ & - & $\infty$ & - & - & กิ \\
\hline \multirow{3}{*}{ ¿̊) } & 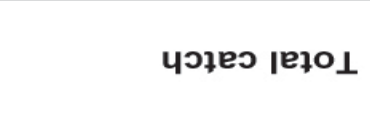 & ষ্ণি & $\begin{array}{l}\text { ᄋ్ } \\
\text { \&্ণ }\end{array}$ & 0 & 0 & 암 & 웅 & $\begin{array}{l}\stackrel{\circ}{0} \\
\stackrel{0}{\sim}\end{array}$ & $\frac{\text { 잉 }}{6}$ & ○ & ○ & 怘 & ষ্ণী & 용 & 0 & 0 & 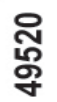 \\
\hline & †әu jo uo!̣esədo & N & 饮 & $\circ$ & 0 & $\sim$ & ㅇ & $\stackrel{\sim}{\sim}$ & $\infty$ & o & 0 & 요 & $m$ & の & 0 & ○ & 今ે \\
\hline & $d !$ & $\checkmark$ & $\lesssim$ & 0 & 0 & $\leftarrow$ & $\stackrel{m}{\check{2}}$ & $\stackrel{m}{\varpi}$ & 움 & ○ & 0 & 음 & - & $\bullet$ & 0 & 0 & 우 \\
\hline \multirow{3}{*}{ চั } & 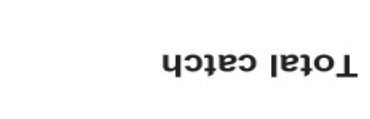 & ర్లి & $\begin{array}{l}\text { 尺े } \\
\text { Оे }\end{array}$ & 유 & 0 & 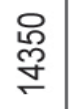 & 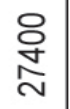 & $\begin{array}{l}8 \\
\stackrel{8}{N} \\
\sim\end{array}$ & $\begin{array}{l}\stackrel{\circ}{0} \\
\stackrel{0}{\forall}\end{array}$ & 0 & ○ & 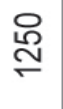 & 0 & $\begin{array}{l}8 \\
\text { \& } \\
\infty\end{array}$ & 各 & 0 & ్ా \\
\hline & łəu jo uo!̣esədo & $\simeq$ & ケ & $m$ & 0 & 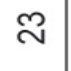 & ๗ & ஜ & $\stackrel{n}{N}$ & ○ & ○ & $m$ & 0 & 으 & $\infty$ & 0 & 요 \\
\hline & d! & $\omega$ & 요 & $\leftarrow$ & 0 & $\mp$ & $\underset{\ulcorner}{ }$ & 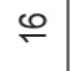 & ా্ল & 0 & 0 & $\sim$ & o & $\infty$ & $\nabla$ & 0 & ஜூర \\
\hline \multicolumn{2}{|l|}{ 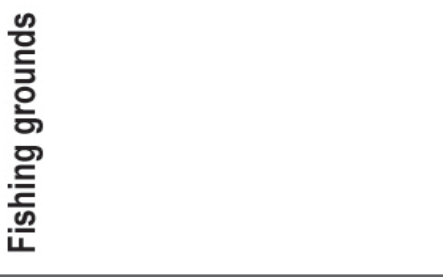 } & 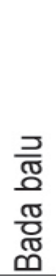 & 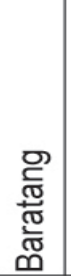 & 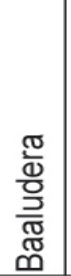 & 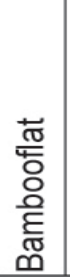 & 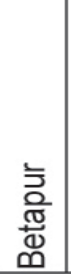 & 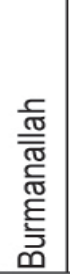 & 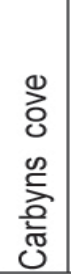 & 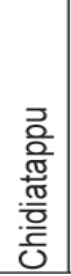 & $\begin{array}{l}\text { 등 } \\
\frac{0}{\overline{0}} \\
\frac{0}{0} \\
\frac{1}{0}\end{array}$ & 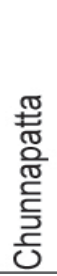 & 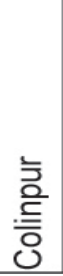 & 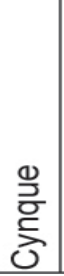 & 言 & 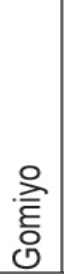 & 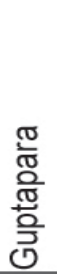 & $\begin{array}{l}\text { 응 } \\
\text { 응 } \\
\text { 종 }\end{array}$ \\
\hline
\end{tabular}




\begin{tabular}{|c|c|c|c|c|c|c|c|c|c|c|c|c|c|c|c|c|c|c|c|c|c|c|c|}
\hline$\stackrel{\infty}{\text { ஸे }}$ & 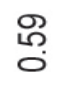 & 용 & $\stackrel{N}{\sigma}$ & 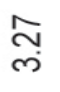 & $\frac{0}{i n}$ & $\begin{array}{l}\stackrel{\infty}{\circ} \\
\stackrel{m}{m}\end{array}$ & 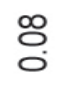 & $\stackrel{2}{2}$ & స్తి & $\stackrel{g}{\circ}$ & 응 & ले & $\stackrel{\infty}{\infty}$ & $\underset{\forall}{\stackrel{N}{*}}$ & ণ & $\stackrel{+\infty}{-}$ & 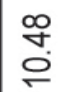 & ઼ָ & $\begin{array}{l}@ \\
\infty \\
0\end{array}$ & $\stackrel{\infty}{\circ}$ & 禺 & $\frac{\sim}{\sim}$ & 응 \\
\hline $\begin{array}{l}8 \\
\text { గn } \\
\qquad\end{array}$ & గ్లి & $\begin{array}{l}\text { ల్లో } \\
\text { 命 }\end{array}$ & : & $\begin{array}{l}\text { స్ } \\
\text { స్ }\end{array}$ & థా & $\begin{array}{l}\stackrel{\circ}{\circ} \\
\stackrel{్}{0}\end{array}$ & 尽 & ֻ & 아 & 忍 & 음 & 웅 & 足 & 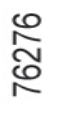 & 유 & $\begin{array}{l}\text { గొ } \\
\stackrel{N}{\mathscr{N}}\end{array}$ & $\begin{array}{l}8 \\
\& \\
00 \\
0\end{array}$ & 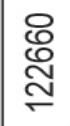 & $\begin{array}{l}\stackrel{2}{\circ} \\
\stackrel{0}{\varpi}\end{array}$ & $\underset{\overbrace{}}{\stackrel{丶}{\rightleftharpoons}}$ & 옹 & 弚 & 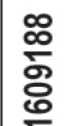 \\
\hline$\mp$ & $\stackrel{\infty}{\sim}$ & প্ল & N & 음 & $\stackrel{5}{n}$ & 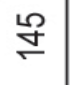 & $\sim$ & $m$ & $\sim$ & in & $\sim$ & مـ & 5 & $\stackrel{Ð}{\simeq}$ & $\leftarrow$ & ฮ & $\stackrel{\sim}{\approx}$ & న్ న్ & ষ & ిలి & $\underset{\sim}{ }$ & $\widetilde{\varrho}$ & ஜ্ট \\
\hline$\sim$ & $\cong$ & $\stackrel{2}{\square}$ & $m$ & గొ & б & छ & $\leftarrow$ & $m$ & $\sim$ & D্থ & 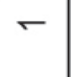 & $m$ & m & ఠర & $\leftarrow$ & ఉా & 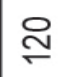 & $\stackrel{ }{\simeq}$ & $\stackrel{\text { D }}{\circ}$ & & $\stackrel{\varphi}{\circ}$ & $\check{\forall}$ & م \\
\hline$\stackrel{8}{\stackrel{8}{ }}$ & 유 & ஜ্ণ & 음 & ণి & 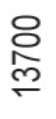 & 員 & 0 & 0 & ర్లి & 志 & 0 & ిొ & ర్రి & 导 & 0 & م્స & 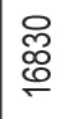 & 음 & 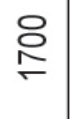 & $\underset{\infty}{\infty}$ & ర్రి & ৪্ণ & 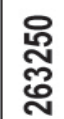 \\
\hline مـ & $r$ & $\bar{\sim}$ & $m$ & $\lesssim$ & 아 & ஜ্ల & 0 & 0 & - & లి & 0 & $\sigma$ & $\underset{\perp}{\Xi}$ & F & 0 & $\approx$ & ఖ్ల & 뉴 & $\mp$ & $\stackrel{20}{\square}$ & $\infty$ & $\cong$ & ల్ల \\
\hline$m$ & $\nabla$ & () & - & $\underset{\ulcorner}{ }$ & ㄱ & $\stackrel{m}{\longleftarrow}$ & 0 & 0 & - & $\simeq$ & 0 & 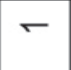 & $\infty$ & ㄱ & 0 & $\simeq$ & ㄱ & $\mathscr{N}$ & $\nabla$ & 으 & 6 & $\infty$ & ষ్ల \\
\hline $\begin{array}{l}\text { 으 } \\
\infty\end{array}$ & 욤 & 요 & 응 & 워 & $\begin{array}{l}\varnothing \\
\varnothing \\
\varnothing\end{array}$ & 융 & 0 & ని & 유 & 옹 & 0 & 0 & 윾 & $\begin{array}{l}\infty \\
\infty \\
\infty \\
\stackrel{\infty}{\sim}\end{array}$ & 0 & 유 & 유 & $\begin{array}{l}\text { 유 } \\
\text { ్ㅠ }\end{array}$ & ণ্ণি & $\frac{8}{6}$ & 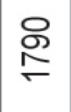 & $\begin{array}{l}\infty \\
\infty \\
\text { ల్ }\end{array}$ & $\begin{array}{l}\text { 广్ } \\
\text { ర్ల } \\
\text { ర్ల }\end{array}$ \\
\hline$\nabla$ & $\sim$ & $\stackrel{m}{\sim}$ & $m$ & $\underset{\leftarrow}{ }$ & 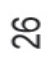 & D & 0 & 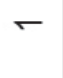 & - & $\mp$ & 0 & 0 & $\mp$ & ল & 0 & $\mp$ & ల్ల & ా্ల & $\simeq$ & 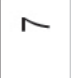 & م & $\cong$ & م \\
\hline$m$ & - & $r$ & - & $\infty$ & $\stackrel{20}{\sim}$ & $\stackrel{m}{\square}$ & 0 & $\sigma$ & - & $\infty$ & 0 & 0 & $\infty$ & $\approx$ & 0 & 0 & 음 & 임 & 10 & 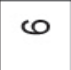 & $\nabla$ & $\sigma$ & ్ㅏㄹ \\
\hline 음 & 只 & 유 & 0 & 용 & $\begin{array}{l}\stackrel{8}{ } \\
\infty \\
0 \\
0\end{array}$ & 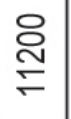 & 0 & రి & 0 & 응 & 0 & 0 & $\frac{}{\frac{}{}}$ & $\begin{array}{l}\text { 옹 } \\
\infty\end{array}$ & 0 & ర్లి & 웡 & $\begin{array}{l}\text { ㅇ } \\
\text { 品 } \\
\text { లె }\end{array}$ & $\frac{8}{\stackrel{+}{+}}$ & ণ্ & 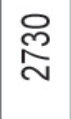 & 尽 & 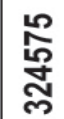 \\
\hline$\sim$ & N & $\leftarrow$ & 0 & 으 & ले & প్ల & 0 & 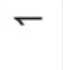 & 0 & 0 & 0 & 0 & $\stackrel{m}{\leftarrow}$ & స & 0 & の & 8 & $\mathfrak{N}$ & N & $m$ & 0 & $\sim$ & : \\
\hline- & - & $\sigma$ & 0 & $\omega$ & $\stackrel{d}{\sim}$ & $\mp$ & 0 & $\sigma$ & 0 & 0 & 0 & 0 & $\infty$ & $\stackrel{2}{\sim}$ & 0 & 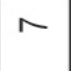 & ల్ల & ల్ల & $\sim$ & $\sim$ & $m$ & $\nabla$ & న్స \\
\hline 0 & 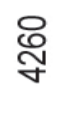 & 0 & 0 & 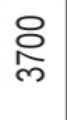 & $\begin{array}{l}\text { ర్ } \\
\text { స్ }\end{array}$ & $\begin{array}{l}\text { ஜ } \\
\text { ᄋ }\end{array}$ & 0 & 0 & 0 & 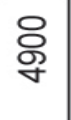 & 0 & 음 & ঙ্ণ & 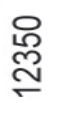 & ద్టి & 오 & 유 & $\frac{\circ}{i n}$ & ৪্ণ & $\underset{\sim}{\stackrel{尺}{N}}$ & 응 & ষ্ণ & 옹 \\
\hline 0 & $\simeq$ & 0 & 0 & () & ఉా & 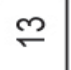 & 0 & 0 & 0 & $r$ & 0 & $\sigma$ & $m$ & $\stackrel{\infty}{\leftarrow}$ & $\sigma$ & $\infty$ & $\stackrel{8}{y}$ & $\bar{m}$ & $\sim$ & م) & 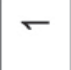 & ㅇ & శ్ల్గ \\
\hline 0 & 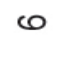 & 0 & 0 & 10 & ㄱ & $\infty$ & 0 & 0 & 0 & $\forall$ & 0 & 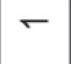 & $m$ & $\infty$ & $\sigma$ & $\nabla$ & $\stackrel{\llcorner}{\sim}$ & $\mathscr{\varphi}$ & $\sim$ & 10 & $\sigma$ & $\cong$ & : \\
\hline 0 & 0 & : & চి & $\begin{array}{l}8 \\
\stackrel{0}{N} \\
\stackrel{N}{N}\end{array}$ & ষ্ণি & $\frac{8}{80}$ & ণ্ণ & 으 & 0 & స్ల్ల & 윰 & $\frac{8}{8}$ & ల్లి & ్ి & 0 & $\frac{8}{\stackrel{8}{4}}$ & $\begin{array}{l}\text { 유 } \\
\text { م }\end{array}$ & $\begin{array}{l}8 \\
\stackrel{0}{N} \\
\text { N }\end{array}$ & 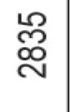 & 0 & 율 & 응 & 융 \\
\hline 0 & 0 & $\nabla$ & - & & ㄱ & ఇ & $\sim$ & - & 0 & 으 & $\sim$ & $m$ & 은 & $\infty$ & 0 & $\cong$ & ஜ & 뇨 & $\cong$ & 0 & $\nabla$ & $\approx$ & 苍 \\
\hline 0 & 0 & $\sim$ & - & 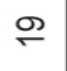 & $\simeq$ & 읻 & $\leftarrow$ & $\leftarrow$ & 0 & م) & $\leftarrow$ & $\leftarrow$ & 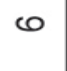 & $\nabla$ & 0 & ما & ㅁ & $\stackrel{\sim}{\sim}$ & 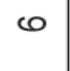 & 0 & $\sim$ & $\infty$ & స్ల \\
\hline 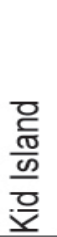 & 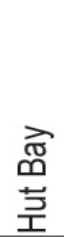 & 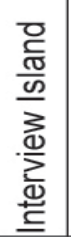 & 袞 & $\begin{array}{l}\frac{\overline{0}}{\bar{c}} \\
\frac{\overline{0}}{0} \\
\frac{0}{0} \\
\overline{0} \\
\end{array}$ & 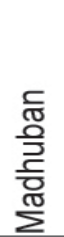 & 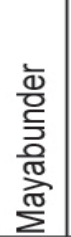 & 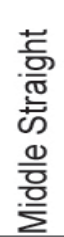 & 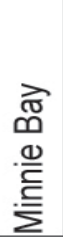 & 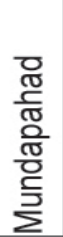 & 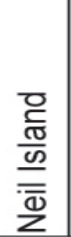 & 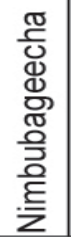 & 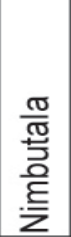 & 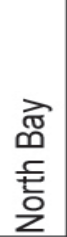 & 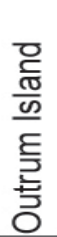 & 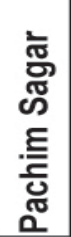 & 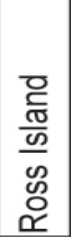 & 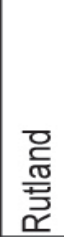 & 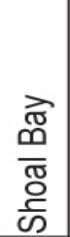 & 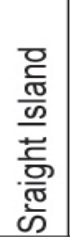 & 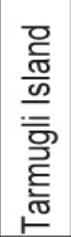 & 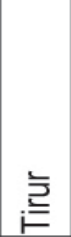 & 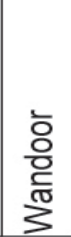 & $\begin{array}{l}\text { 퓨 } \\
\text { 으 }\end{array}$ \\
\hline
\end{tabular}


TABLE - 4: Month wise data regarding trip, operation and fish catch from four FLC for the period 20142018

\begin{tabular}{l|c|c|c|c|c|c}
\hline $\begin{array}{l}\text { Name of the } \\
\text { Month }\end{array}$ & $\begin{array}{c}\text { Total } \\
\text { Trip }\end{array}$ & $\begin{array}{c}\text { Trip } \\
\text { Av/month }\end{array}$ & $\begin{array}{c}\text { Gear } \\
\text { Operation }\end{array}$ & $\begin{array}{c}\text { Gear } \\
\text { Operation } \\
\text { Av/month }\end{array}$ & $\begin{array}{c}\text { Fish } \\
\text { Catch } \\
\text { (in ton) }\end{array}$ & $\begin{array}{c}\text { Av/month } \\
\text { (in ton) }\end{array}$ \\
\hline January & 90 & 18.0 & 90 & $18.0 \pm 3.5$ & 109.4 & $21.9 \pm 4.0$ \\
\hline February & 97 & 19.4 & 114 & $22.8 \pm 2.4$ & 134.3 & $26.9 \pm 3.5$ \\
\hline March & 100 & 20.0 & 94 & $18.8 \pm 3.3$ & 107.5 & $21.5 \pm 3.3$ \\
\hline April & 82 & 16.4 & 105 & $21.0 \pm 2.1$ & 151.4 & $30.3 \pm 4.1$ \\
\hline May & 88 & 17.6 & 115 & $23.0 \pm 1.8$ & 125.0 & $25.0 \pm 4.0$ \\
\hline June & 98 & 19.6 & 128 & $25.6 \pm 6.3$ & 149.7 & $29.9 \pm 7.9$ \\
\hline July & 105 & 21.0 & 165 & $33.0 \pm 3.0$ & 179.8 & $36.0 \pm 9.3$ \\
\hline August & 96 & 19.2 & 137 & $27.4 \pm 3.2$ & 150.0 & $30.0 \pm 3.5$ \\
\hline September & 93 & 18.6 & 123 & $24.6 \pm 2.5$ & 137.0 & $27.4 \pm 3.9$ \\
\hline October & 86 & 17.2 & 98 & $19.6 \pm 2.4$ & 122.7 & $24.5 \pm 1.9$ \\
\hline November & 16.4 & 98 & $19.6 \pm 2.1$ & 122.5 & $24.5 \pm 2.4$ \\
\hline December & 86 & 112 & $22.4 \pm 3.7$ & 120.0 & $24.0 \pm 4.7$ \\
\hline
\end{tabular}

and Non-PFZ areas.

The length-weight relationships (LWR) of fishes are very important in fishery biology with the various developmental events in life such as metamorphosis, growth and on-set of maturity ${ }^{39}$. After the receipt of the satellite picture, the same is printed and disseminated amongst the fishermen to enhance their fish catch by indicating with Lat \& Long and reducing the scouting time.

Identification of Potential Fishing Zones involves an understanding of oceanic processes and interaction of hydro-biological parameters ${ }^{8}$. Each fishing operation executes a unique harvesting technique and thus varies in terms of number of crew, duration of fishing, depth of operation, type of gear deployed and species/quantity of fish harvested ${ }^{25}$.

\section{Result and Discussion}

It was concluded that the average income generated by vessels operating in the PFZ areas were significantly more than vessels operating in non PFZ areas.
The expenses of fishing operation were also comparatively lower than vessels which operated within PFZ.

The data have been collected for 5 (five) consecutive years (2014 to 2018) from four fish landing centres (FLC) viz Junglighat, Dugnabad, Guptapara and Wandoor in South Andaman. The visits have been carried out at above mentioned four FLCs during the study period. The maximum visits have been occurred during the year 2016 (261) and the minimum visits were done during the year 2018 (181). Altogether 1097 numbers of visits have been occurred with an average of 219 visits per year (Table 1 ). According to the FLC wise visit (Table 1 ), the maximum visits have been done at Junglighat (692) followed by Dugnabad (169), Guptapara (129) and Wandoor (107). Similarly the landings of fishes were observed more at Junglighat FLC (3825.9 ton) followed by Dugnabad (30.4 ton), Guptapara (12.4 ton) and Wandoor (12 ton) during the visiting period.

The total operations of Ring net 1379 were observed 
TABLE - 5: Validation conducted on Ring net operation In and Out of PFZ during the year 2014 to 2018.

\begin{tabular}{l|c|c|c|c|c|c|c}
\hline Year & $\mathbf{2 0 1 4}$ & $\mathbf{2 0 1 5}$ & $\mathbf{2 0 1 6}$ & $\mathbf{2 0 1 7}$ & $\mathbf{2 0 1 8}$ & Total & Average \\
\hline Validations conducted at PFZ & 13 & 3 & 13 & 9 & 11 & 49 & $9.8 \pm 1.9$ \\
\hline Fish catch in PFZ (in tons) & 24.1 & 2.5 & 24.5 & 7.8 & 32.2 & 91.1 & $18.2 \pm 5.6$ \\
\hline Validation conducted at Non-PFZ & 13 & 3 & 13 & 9 & 11 & 49 & $9.8 \pm 1.9$ \\
\hline Fish catch in Non-PFZ (in tons) & 6.8 & 0.7 & 6.9 & 1.7 & 7.8 & 23.8 & $4.8 \pm 1.5$ \\
\hline
\end{tabular}

Length weight relationship of the pelagic fishes captured at PFZ and Non-PFZ:

from two FLC i.e. Junglighat (1322) and Dugnabad (57) amongst four FLC. The total pelagic fishes were captured 1609.2 tons with an average of 321.8 tons / year. It was observed that the fish catch from the above two FLC were 1594.2 tons and 15 tons respectively. The contribution of the gear Ring net was $41.5 \%$ amongst the other gears operated from all four FLCs. The more fish catches of the gear ring net were Scombrids (52.3\%) followed by Sardines (22.4\%), Carangids (21.3\%), Anchovies (2.7\%) and others (1.2\%) (Table-2). Among the scombrids R. kanagurta was observed to be dominant and the catch of other scombrids like Little tuna i.e. Auxis thazard, Yellow fin tuna i.e. Thunnus albacares, Skipjack tuna i.e. Katsuwonus pelamis etc., also observed occasionally at low quantity. Among the clupidae Sardinella albella, Sardinella sirm, Sardinella brachisoma, Herklotsichthys quadrimaculatus etc were observed to be dominant in the landings. The other fishes are Milk fish (Chanos chanos), Silver belly (Lieognothus sp.), Silver buddy (Gerres sp.), Barracuda (Sphyreana sp.), Devil Ray (Manta birostris) (Fig. 2).

According to the yearly study the maximum ring net operations and maximum fish catch occurred during the year 2014 i.e. 298 nos. and 398.06 tons respectively. The minimum gear operation (253) occurred during the year 2017 and minimum fish catch (263.25) observed during the year 2018 (Fig. 3)

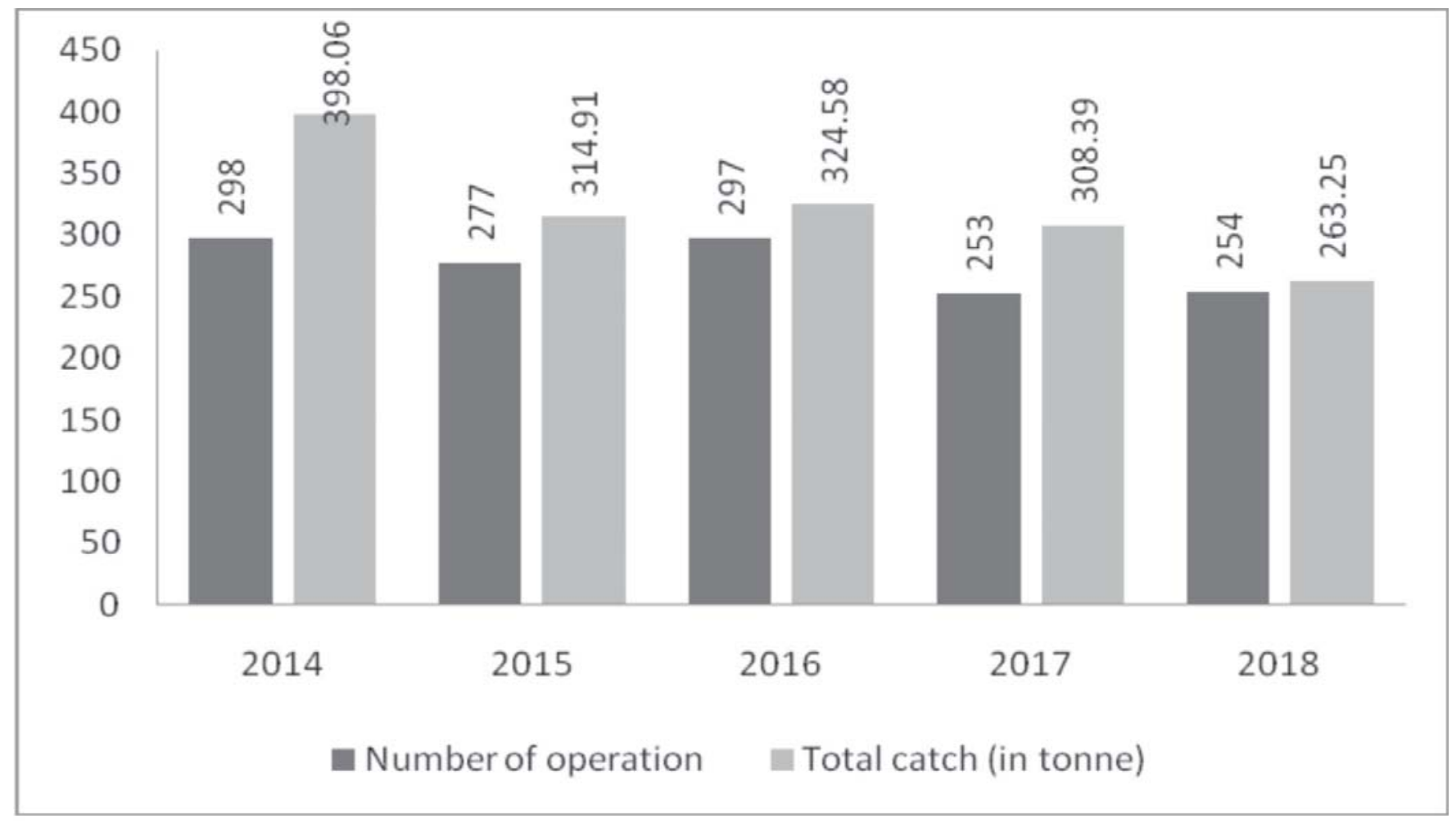

Fig. 3 Diagram showing the year wise (2014-18) boat operation and fish catch in tons 
TABLE - 6 : Length weight of the pelagic fishes captured at PFZ and Non-PFZ:

\begin{tabular}{|c|c|c|c|c|}
\hline \multirow{3}{*}{$\begin{array}{l}\text { Species } \\
\text { Family: CARANGIDAE }\end{array}$} & \multicolumn{2}{|c|}{ Range } & \multicolumn{2}{|c|}{ Mean (mean $\pm \mathrm{SE}$ ) } \\
\hline & \multicolumn{2}{|c|}{ Length (mm) } & \multicolumn{2}{|c|}{ Weight (g) } \\
\hline & & & & \\
\hline Atule mate (PFZ) & $100-260$ & $180.17 \pm 1.63$ & $9-175$ & $74.78 \pm 1.90$ \\
\hline Atule mate (Non - PFZ) & $102-243$ & $168.73 \pm 1.34$ & $10-163$ & $61.93 \pm 1.51$ \\
\hline Decapterus russelli (PFZ) & $108-202$ & $160.65 \pm 0.99$ & $12-78$ & $38.62 \pm 0.68$ \\
\hline Decapterus russelli (Non-PFZ) & $130-197$ & $161.43 \pm 0.68$ & $12-72$ & $38.03 \pm 0.51$ \\
\hline Family: CLUEPIDAE & & & & \\
\hline Sardinella albella (PFZ) & $120-166$ & $141.61 \pm 0.46$ & $14-41$ & $25.40 \pm 0.27$ \\
\hline Sardinella albella (Non-PFZ) & $120-162$ & $139.35 \pm 0.42$ & $14-36$ & $23.78 \pm 0.25$ \\
\hline Sardinella sirm (PFZ) & $185-260$ & $219.92 \pm 0.70$ & $59-181$ & $108.92 \pm 1.12$ \\
\hline Sardinella sirm (Non-PFZ) & $136-256$ & $219.07 \pm 0.75$ & $22-177$ & $108.43 \pm 1.22$ \\
\hline Herklotsichthys quadrimaculatus (PFZ) & $83-145$ & $119.54 \pm 0.56$ & $4-32$ & $16.51 \pm 0.26$ \\
\hline Herklotsichthys quadrimaculatus (Non-PFZ) & $105-150$ & $121.6 \pm 0.38$ & $10-28$ & $16.54 \pm 0.18$ \\
\hline Family: SCOMBRIDE & & & & \\
\hline Rastrelliger kanagurta (PFZ) & $145-320$ & $250.6 \pm 1.09$ & $32-410$ & $188.37 \pm 2.45$ \\
\hline Rastrelliger kanagurta (Non-PFZ) & $142-308$ & $233.25 \pm 1.37$ & 29-361 & $152.14 \pm 2.78$ \\
\hline
\end{tabular}

\section{Fishing ground:}

Altogether 39 fishing grounds have been identified and 1575 fishing ground trips have been carried out with 3069 fishing operation during the study period by the ring netters (Table-3). The fishermen ventured repeatedly at Potential Fishing ground by using PFZ forecast which is received from INCOIS, Hyderabad. The maximum fishing ground trips (225) and maximum number of operation (481) occurred around the Coast of Havelock Island. The maximum fish catch (249.9 tons) occurred around the Coast of Havelock Island (15.5\%) followed by Rutland (10.5\%), Chidiatappu (8.0\%), Shoal Bay (7.6\%), Baratang (7.0\%), Carbyns cove (6.0\%), Burmanallah (5.2\%), Madhuban (5.2\%), Out-drum Island (4.7\%), Mayabunder
(4.0\%), Colinpur (3.3\%), Long Island (3.3\%), Wandoor (2.1\%) etc and minimum fish catch was observed at the coast of Pachim Sagar (0.02\%).

The month wise FLC visits, gear operation and fish catch during the study period (January 2014 to December 2018) have been analyzed. The maximum number of FLC visit was observed in the month of July (105) with an average of 21 and minimum was observed during December (80) with an average of 16 . The gear ring net operation was observed maximum in July (165) with an average of 33 and minimum during January (90) with average of 18 . The fish catch found maximum (179.8 tons) during the month of July with an average of 36 tons and minimum catch observed on March (107.5 tons) with an 
TABLE - 7: The composition of fishes under different class of interval in PFZ and Non-PFZ.

\begin{tabular}{|c|c|c|c|c|c|c|c|c|c|c|c|c|}
\hline \multirow{3}{*}{$\begin{array}{l}\text { Length } \\
\text { Classes } \\
(\mathrm{mm})\end{array}$} & \multicolumn{2}{|c|}{ Atule mate } & \multicolumn{2}{|c|}{$\begin{array}{l}\text { Decapterus } \\
\text { russelli }\end{array}$} & \multicolumn{2}{|c|}{$\begin{array}{l}\text { Sardinella } \\
\text { albella }\end{array}$} & \multicolumn{2}{|c|}{$\begin{array}{l}\text { Sardinella } \\
\text { sirm }\end{array}$} & \multicolumn{2}{|c|}{$\begin{array}{l}\text { Herklotsichthys } \\
\text { quadrimaculatus }\end{array}$} & \multicolumn{2}{|c|}{$\begin{array}{r}\text { Rastrelliger } \\
\text { kanagurta }\end{array}$} \\
\hline & \multicolumn{2}{|c|}{ (\%) } & \multicolumn{2}{|c|}{ (\%) } & \multicolumn{2}{|c|}{ (\%) } & \multicolumn{2}{|c|}{ (\%) } & \multicolumn{2}{|c|}{ (\%) } & \multicolumn{2}{|r|}{ (\%) } \\
\hline & PFZ & $\begin{array}{l}\text { Non- } \\
\text { PFZ }\end{array}$ & PFZ & $\begin{array}{l}\text { Non- } \\
\text { PFZ }\end{array}$ & PFZ & $\begin{array}{l}\text { Non- } \\
\text { PFZ }\end{array}$ & PFZ & $\begin{array}{l}\text { Non- } \\
\text { PFZ }\end{array}$ & PFZ & $\begin{array}{l}\text { Non- } \\
\text { PFZ }\end{array}$ & PFZ & $\begin{array}{l}\text { Non- } \\
\text { PFZ }\end{array}$ \\
\hline $81-100$ & & & & & & & & & 4.8 & 0.0 & & \\
\hline $101-120$ & 5.5 & 5.7 & 2.8 & 0.0 & 0.8 & 0.6 & & & 45.7 & 49.4 & & \\
\hline $121-140$ & 19.1 & 23.4 & 15.0 & 4.5 & 44.4 & 54.3 & 0.0 & 0.6 & 47.7 & 48.6 & & \\
\hline $141-160$ & 16.7 & 20.3 & 23.2 & 49.0 & 53.1 & 44.8 & 0 & 0 & 1.8 & 2.0 & 0.8 & 5.0 \\
\hline $161-180$ & 10.9 & 7.4 & 48.6 & 39.1 & 1.6 & 0.3 & 0.0 & 0.3 & & & 0.6 & 4.6 \\
\hline $181-200$ & 6.7 & 17.8 & 8.8 & 7.4 & & & 11.2 & 7.4 & & & 2.3 & 2.7 \\
\hline $201-220$ & 17.8 & 19.9 & 1.7 & 0.0 & & & 37.9 & 42.6 & & & 13.0 & 23.0 \\
\hline $221-240$ & 14.3 & 5.4 & & & & & 48.2 & 45.4 & & & 15.7 & 20.0 \\
\hline $241-260$ & 9.0 & 0.4 & & & & & 2.7 & 3.7 & & & 27.1 & 21.1 \\
\hline $261-280$ & & & & & & & & & & & 30.9 & 18.4 \\
\hline $281-300$ & & & & & & & & & & & 8.7 & 4.3 \\
\hline $301-320$ & & & & & & & & & & & 0.9 & 0.8 \\
\hline
\end{tabular}

average of 21.5 tons (Table 4).

\section{Validation for PFZ advisories:}

PFZ forecast disseminated to the Ring netters and Validated during the study period. A total of 49 validations for PFZ advisories carried out at each PFZ and Non- PFZ with an average of 9.8 operations /year. The fishes captured from the PFZ were 91.1 tons with an average of $18.2 \pm 5.6$ tons / year. Similarly the fishes captured from the Non PFZ were 23.8 tons with an average of $4.5 \pm 1.5$ tons /year. (Table.5). The validation of PFZ advisories have inferred significant increase in fish catch along northwest coast of Gujarat ${ }^{10,25,34,35}$ and all over the country ${ }^{6}$. The annual exploitable fisheries of Andaman and Nicobar Islands are estimated to be 1.48 lakh $t$ of which a meagre $22 \%$ is harvested currently. Potential Fishing Zone (PFZ) forecasts based on remotely sensed chlorophyll concentration and sea surface temperature were applied for harvesting the unexploited marine fishery resources ${ }^{17}$.

The number of fish samples Atule mate collected from the FLC which captured at PFZ and Non PFZ were 670 and 680 respectively. The number of fish samples of Decapterus russelli, Sardinella albella, Sardinella sirm, Herklotsichthys quadrimaculatus and Rastrelliger kanagurta collected from the FLC which captured at PFZ were 354, 367, 367, 396 and 645 respectively. The number of same species samples collected from Non- PFZ were $353,357,350,350$ and 625 respectively.

Fish samples of six pelagic species viz., Atule mate, Decapterus russelli under the family Carangidae, Herklotsichthys quadrimaculatus, Sardinella albella and Sardinella sirm under the family Cluepidae and Rastrelliger kanagurta under the family Scombridae have 

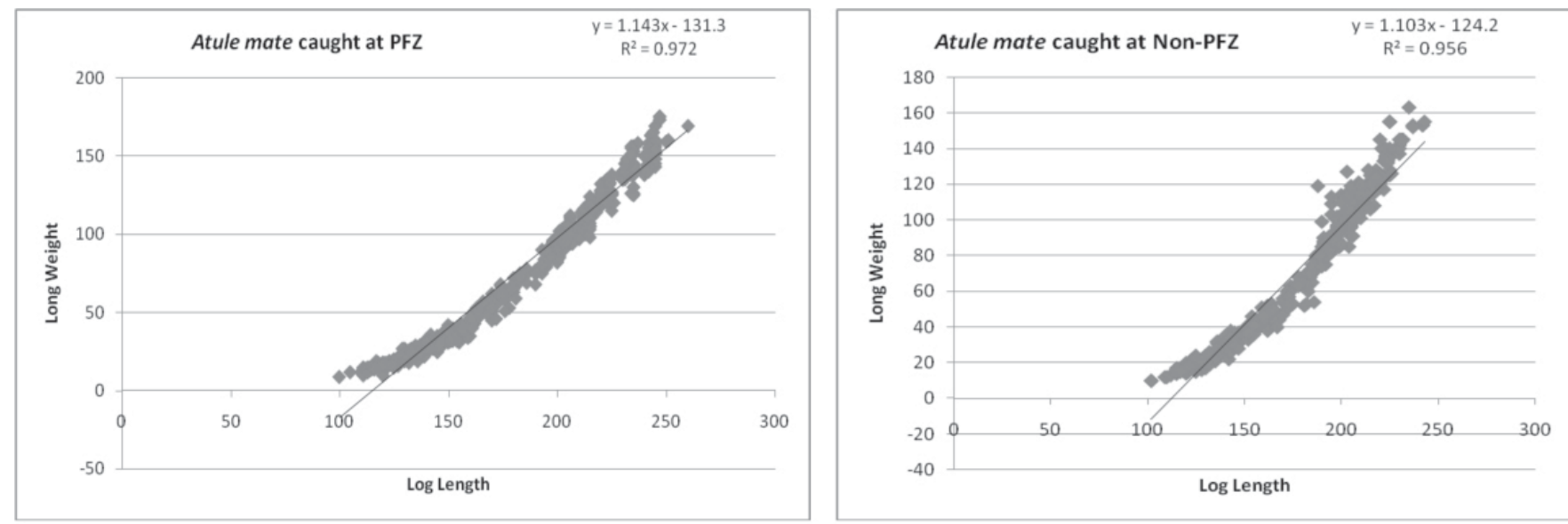

Fig. 4. LWR of Atule mate at in \& outside of PFZ

been collected from the landing centres, which were captured from PFZ and Non-PFZ of Andaman Islands and Length frequency of the fish has been carried out. Details with respect to the sample size $(n)$, the mean ( \pm SE) length and weight are summarized in the Table 6.

The length of Atule mate at PFZ and Non-PFZ were ranging from $100-260 \mathrm{~mm}$ with a mean length of $180.17 \pm$ $1.63 \mathrm{~mm}$ and $102-243 \mathrm{~mm}$ with mean length $168.73 \pm$ $1.34 \mathrm{~mm}$ respectively. The weight of Atule mate at PFZ and Non-PFZ were ranging from $9-205 \mathrm{~g}$ with a mean weight $75.13 \pm 1.91 \mathrm{~g}$ and ranging from 10-182 with mean weight $62.18 \pm 1.53 \mathrm{~g}$ respectively. The length of $D$. russelli at PFZ and Non-PFZ were ranging from 130-201 mm with a mean length of $165.57 \pm 1.04 \mathrm{~mm}$ and $143-193 \mathrm{~mm}$ with mean length $159.49 \pm 0.56 \mathrm{~mm}$ respectively. The weight of Decapterus russelli at PFZ and Non-PFZ were ranging from $15-81 \mathrm{~g}$ with a mean weight $42.15 \pm 0.86 \mathrm{~g}$ and ranging from $25-80$ with mean weight $37.42 \pm 0.56 \mathrm{~g}$ respectively.

The anchovy is an important food source for almost each and every predatory fishes and is also extremely

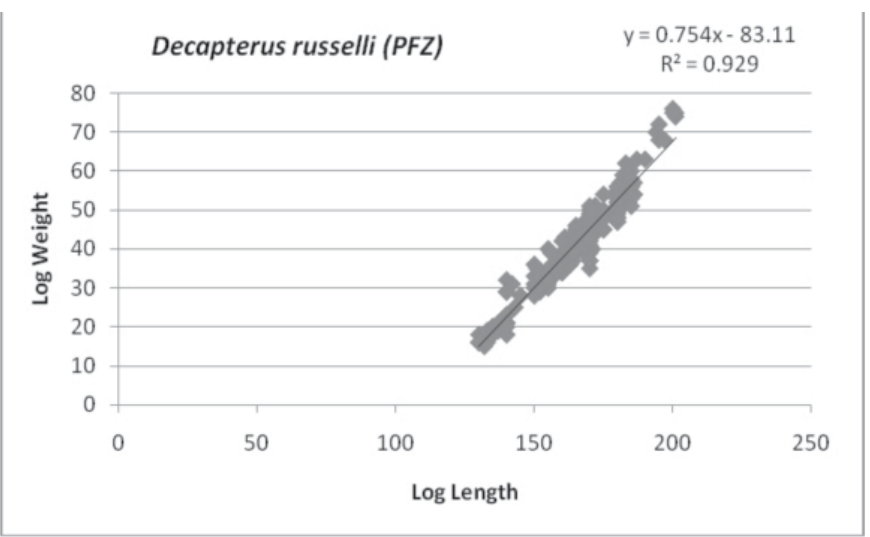

significant to oceanic birds and mammals. Like various fish species of Sardinella, Decapterus and anchovies commonly form schools of similar fish species and uniform size, swimming in a dense formation maintaining an approximately uniform course and speed of swimming. The fish Decapterus russelli is generally coastal schooling fish, are found in the coastal waters between 30-170 m depth. Sardinella species are generally coastal, schooling fishes and abundant richly in warmer waters. There is also accumulation of other fishes, which are attracted to light. Such accumulation can be composed of many fish species and of different sizes whose behaviour is not often uniform.

The length of Sardinella albella, Sardinella sirm and Herklotsichthys quadrimaculatus under the family Cluepidae captured at PFZ were ranging from 121-166, $185-260$ and $83-145 \mathrm{~mm}$ with mean length of $141.0 \mathrm{~mm}$, $219.9 \mathrm{~mm}$ and $119.5 \mathrm{~mm}$ and the weight ranging from $13-41 \mathrm{~g}, 59-181 \mathrm{~g}$ and $4-32 \mathrm{~g}$ with mean weight of $25.2 \mathrm{~g}$, $108.9 \mathrm{~g}$ and $16.5 \mathrm{~g}$ respectively. The length of the same species captured at Non-PFZ were ranging from 120-162

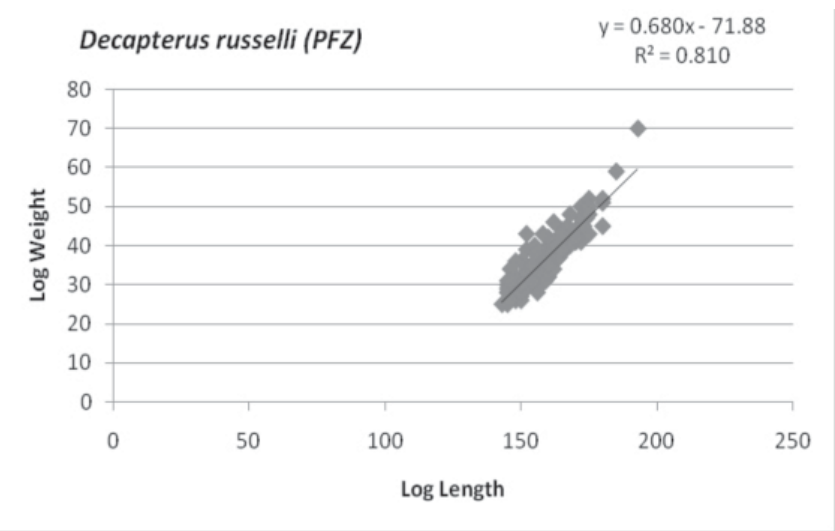



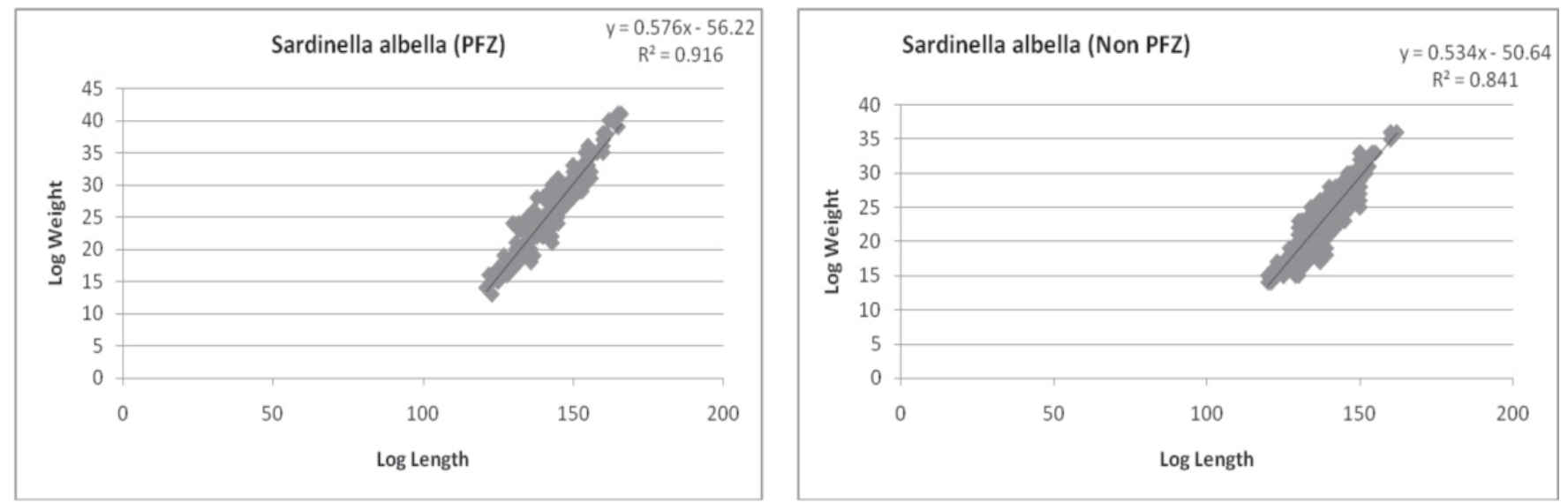

Fig. 6. LWR of Sardinella albella at in \& outside of PFZ

$\mathrm{mm}, 136-256$ and $105-150 \mathrm{~mm}$ with a mean length of $138.2 \mathrm{~mm}, 219.1 \mathrm{~mm}$ and $121.6 \mathrm{~mm}$ and the weight ranging from $14-36 \mathrm{~g}, 22-177 \mathrm{~g}$ and $10-28 \mathrm{~g}$ with mean weight of $23.2 \mathrm{~g}, 108.4 \mathrm{~g}$ and $16.5 \mathrm{~g}$ respectively.

The length of Rastrelliger kanagurta at PFZ and Non-PFZ were ranging from $145-320 \mathrm{~mm}$ with a mean length of $250.6 \pm 1.09 \mathrm{~mm}$ and $142-308 \mathrm{~mm}$ with mean length $233.25 \pm 1.37 \mathrm{~mm}$ respectively. The weight of $R$. kanagurta at PFZ and Non-PFZ were ranging from 32$410 \mathrm{~g}$ with a mean weight $188.37 \pm 2.45 \mathrm{~g}$ and ranging from 29-361 with mean weight $152.14 \pm 2.78 \mathrm{~g}$ respectively (Table-6).

The total of length cattegories were observed from the species Atule mate, Decapterus russelli, Sardinella albella, Sardinella sirm, Herklotsichthys quadrimaculatus and Rastrelliger kanagurta were 9 (81-100 to 241-260), 5 (121-140 to 201-220),4(101-120 to 161-180), 6 (121-140 to 241-260), 4 (81-100 to 141-160), and 9 (141-160 to 301$320)$ respectively. The length category $121-140$ was the most representating class with 128 specimens (19.1\%) at PFZ and 159 specimens (23.7\%) at Non-PFZ respectively from the Atule mate. It has been observed from other species Decapterus russelli, Sardinella albella, Sardinella sirm, Herklotsichthys quadrimaculatus and Rastrelliger kanagurta which collected from the PFZ were class 161-180 mm with 172 specimens (48.6\%), 141$160 \mathrm{~mm}$ with 195 specimens (53.1\%), 221-240 mm with 177 specimens (48.2\%), 121-140 mm with 189 specimens (47.7\%) and 261-280 with 199 specimens (30.9\%) respectively. The same species were collected at the NonPFZ were 141-160 with 173 specimen (49.0\%), 121-140 with 194 specimens (53.3\%), 221-240 with 159 (45.4\%), 101-120 with 173 (49.4\%) and 201-220 with 144 specimens (23\%) respectively (Table 7).

\section{Length frequency of the pelagic fishes from PFZ and} Non- PFZ :

The log converted values of length $(X)$ and weight $(Y)$ are depicted in scatter diagrams and length frequency for all the species above mentioned. Length weight relationships (LWR) were illustrated for all the species above mentioned. The $\mathrm{R}^{2}$ value of Atule mate, Decapterus russelli, Sardinella albella, Sardinella sirm, Herklotsichthys quadrimaculatus and Rastrelliger kanagurta were corresponded at PFZ were 0.972,
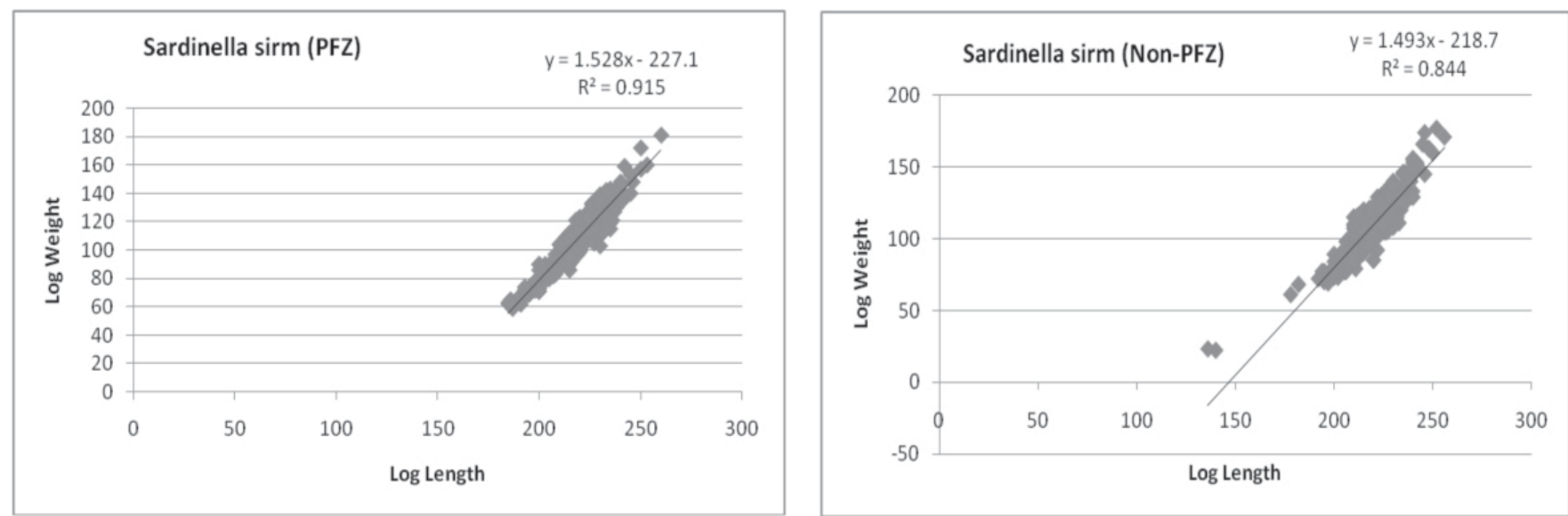

Fig. 7. LWR of Sardinella sirm at in \& outside of PFZa 

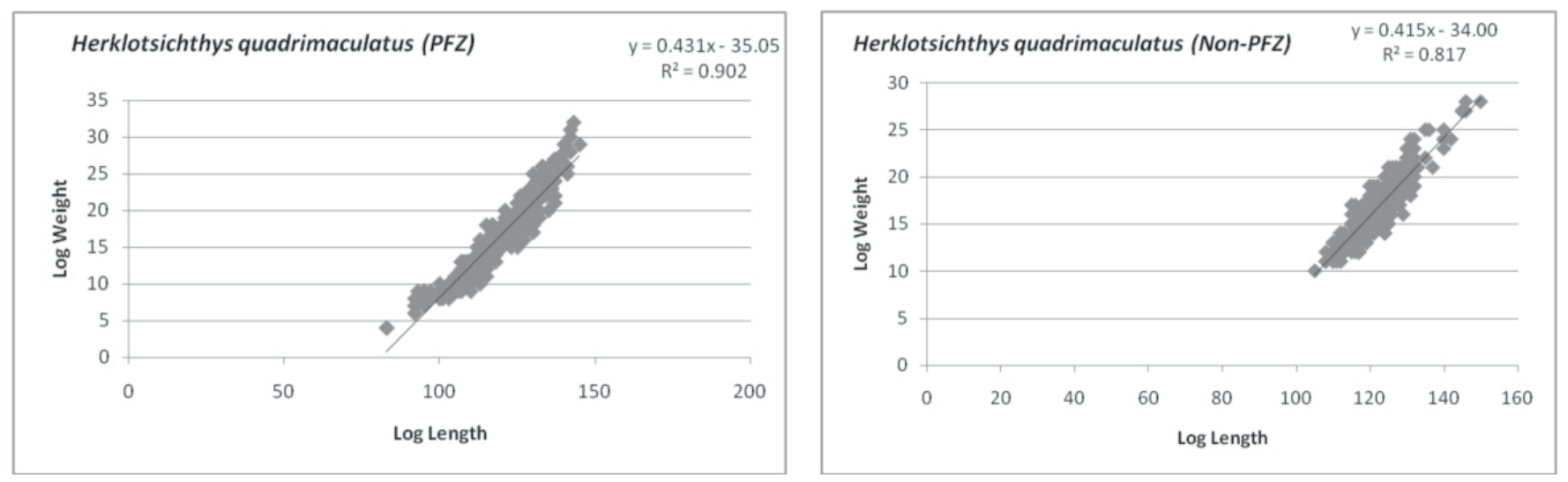

Fig. 8. LWR of Herklotsichthys quadrimaculatus at in \& outside of PFZ

$0.929,0.916,0.915,0.902$ and 0.926 subsequently the same value corresponded at Non-PFZ were $0.956,0.810$, $0.841,0.844,0.817$ and 0.893 respectively. The $R^{2}$ value of all the fishes at PFZ were perfectly correlated which could be attributed to the abundance food from the Potential Fishing Zone. The same value at Non-PFZ is showing less due to less food abundance (kindly find in the figures given below (Fig. 4 - Fig. 9).

\section{Hydrographical study:}

A total of 103 sites were covered during the study period at PFZ and 94 sites were covered at Non-PFZ for the collection of water samples and the traditional fishing grounds along the coast of Andaman Islands during the study period. The average depth which the water samples collected at PFZ and Non- PFZ was $44.72 \pm 1.78$ and $45.08 \pm 2.17$ respectively. The collected water samples from various sites have been analysed. There is no much variation in Sea surface temperature both PFZ and NonPFZ. But according the Secch Disk, there are differences in Visibility between PFZ and Non-PFZ due to more density of both the phyto and zoo planktons. The average visibility at PFZ and Non-PFZ were $15.49 \pm 0.28 \mathrm{~m}$ and $16.74 \pm 0.25$ during respectively. There is no much variation in $\mathrm{pH}$ within and outside of the PFZ i.e. $8.0 \pm 0.02$ and $8.05 \pm 0.02$ respectively. The average dissolved oxygen (DO) at PFZ and Non-PFZ were $6.30 \pm 0.05 \mathrm{mg} / \mathrm{lit}$ and 5.94 $\pm 0.06 \mathrm{mg} /$ lit respectively. The average of Alkalinity at PFZ and Non PFZ were $111.16 \pm 1.09 \mathrm{ml} / \mathrm{lit}$ and $113.68 \pm 1.28$ $\mathrm{ml} /$ lit respectively. There were variation in alkalinity between PFZ and Non-PFZ due to water current and Eddies (upwelling and down welling) more at PFZ.

It is well known that the mesoscale, eddies increase the productivity in a stratified coastal atmosphere by upwelling. The seas around the Andaman and Nicobar Islands have been found to have repeated mesoscale eddy activity. Commercial fishing grounds are coinciding with upwelling areas related with cyclonic and anti-cyclonic eddies and also with areas between two adjoining eddies. There are various eddy zones supporting different types of fishing mechanisms and fishes. The current study aimed at identifying the different zones of mesoscale eddies in the Andaman Sea and compares the productivity and fishing activity in each of them ${ }^{2}$.

The fishing ground Havelock Island is covering $15.5 \%$ fish catch amongst all the 39 fishing ground, Since
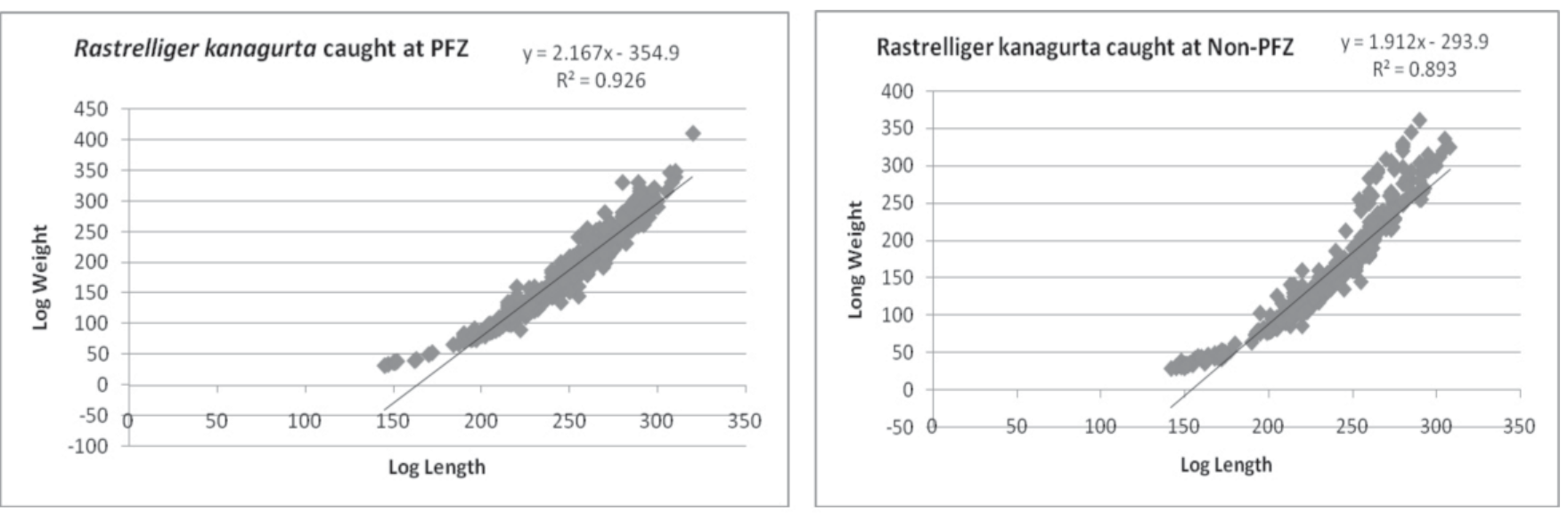

Fig.9. LWR of Rastrelliger kanagurta at in \& outside of PFZ 
the water current running from Hut Bay (Little Andaman) to Havelock Island for a long distance (approximately $150 \mathrm{~km}$ ) transports both the phyto plankton and zoo planktons. The same way, the shoal of pelagic fishes are travelling in straight line from Hut Bay to Havelock for feeding on the abundance of available plankton. The fishing ground landmarks Rutland (10.48\%), Chidiatappu (8.03\%), Shoal Bay (7.62\%), Carbyns cove (5.96\%), Burmanallah (5.17\%), Madhuban (5.16\%) and Colinpur (3.34\%) falls on the same straight line.

A significant increase in total catch by following PFZ forecasts has been documented from $\mathrm{ANI}^{15}$. Validation experiments concludes that satellite based fishing is advantageous in ANI with proven increase in total fish catch in PFZs ${ }^{17}$. The variation of Length and Weight relationship has been observed in both areas. The $\mathrm{R}^{2}$ value of the species Atule mate, Decapterus russelli, Sardinella albella, Sardinella sirm,
Herklotsichthys quadrimaculatus and Rastrelliger kanagurta at PFZ was higher which could be attributed to higher abundance food from the Potential Fishing Zone.

According to the validation the fish catch inside the PFZ gave more CPUE and net profit compared to the outcome of operations in the outside PFZ. Fishing operations carried out on or nearer to dates on which linked SST/Chlorophyll imageries have been received positive outcome. When the gap increases the fish catch within PFZ is likely to decrease unless the features remain more or less in the same fishing location as revealed by the succeeding satellite imagery. There is not much variation in Sea Surface Temperature between PFZ and Non-PFZ. But there are differences between PFZ and NonPFZ in Visibility due to accumulation of both the phytoplankton and zoo planktons. There were variation in alkalinity between PFZ and Non-PFZ due to water current and Eddies (upwelling and down welling) more at PFZ.

\section{References}

1. Advani S, Sridhar A, Namboothri N, Chandi M, Oommen M.A. Emergence and transformation of marine fisheries in the Andaman Islands. Dakshin Foundation and ANET. 2013; p 50.

2. Andfish. Roadmap for the development of fisheries in Andaman and Nicobar Islands. CMFRI and CIFT- Cochin, CIBA- Chennai, CARI- Port Blair and ICAR-Fisheries Division-New Delhi. June 2005; p 89.

3. Anrose A, Sinha mk, Kar Ab. Oceanic tuna resources potential in Andaman and Nicobar waters. In: Proceedings of Brainstorming session on Development of Island Fisheries (Eds. Dam Roy, S., P. Krishnan, K. Sarma and G. George), Central Agricultural Research Institute, Port Blair. 2009; pp. 5-22.

4. Bakun A. "Fronts and Eddies as Key Structures in the Habitat of Marine Fish Larvae: Opportunity, Adaptive Response and Competitive Advantage." Scientia Marina. 2006; 70 (S2): 105-122.

5. Chandi M. The Karen dinghy (Khlee) of the Andaman Islands. 2001; pp. 1-12. ANET Report.

6. Choudhury, SB, Rao KH, Rao MV. Satellite remote sensing for marine resources assessment. Trop. Ecol. 2002; 43(1): 187-202.

7. Cmfri, Annual Report. Central Marine Fisheries Research Institute, Cochin, 2006a. p. 126.

8. Dam Roy S, Grinson George. "Marine Resources of Islands: Status and Approaches for sustainable Exploitation I Conservation with Special Emphasis to Andaman and Nicobar", Indian Journal of Animal Sciences. 2010; 80 (4Suppl.I) : 57-62.

9. Desai PS, Honnegowda H, Kasturirangan K. Ocean research in India: Perspective from space. Curr. Sci. 2000; 78(3): 268-278.

10. Dwivedi RM, Solanki HU, Nayak SR, Gulati DK, Somvanshi VS. Exploration of fishery resources through integration of ocean colour with sea surface temperature: Indian experience. Ind. J. Mar. Sci. 2005; 34(4): 430-440.

11. FAO. Fishery statistics: Capture production. FAO Yearbook. (2002a); 94(1).

12. FAO. The state of world fisheries and aquaculture FAO Fisheries and Aquaculture Department, Food and Agriculture Organization of the United Nations, Rome. (2010a); p. 197.

13. Fridman A.I. (1986).Calculations for fishing gear design. Oxford: Osney Mead.

14. FSI. "National Marine Fisheries Census 2005: Union Territories of Andaman \& Nicobar and Lakshadweep Islands." New Delhi: Ministry of Agriculture, Department of Animal Husbandry, Dairying \& Fisheries, Government of India. 
$2007 ; 177$.

15. George Grinson, Krishnan P, Sarma Kamal, Kirubasankar R, Goutham Bharathi MP, Kaliyamoorthy M, Krishnamurthy V, Kumar ST. Integrated potential fishing zone (IPFZ) forecasts: a promising information and communication technology tool for promotion of green fishing in the islands. Ind. J. Agricult. Econ. 2011; 66(3): 513-519.

16. Grinson George, Kamal Sarma, Goutham Bharathi, Muthuraj Kaliyamoorthy, Pandian Krishnan, Kirubasankar R. Efficacy of different modes in disseminating Potential Fishing Zone (PFZ) forecasts- a case study from Andaman and Nicobar Islands. Indian J. Fish. 2014; 61(1) : 84-87.

17. Grinson-george, Krishnan P, Dam-roy S, Kamal-sarma, Goutham-bharathi MP, Kaliyamoorthy M, Krishnamurthy V, Srinivasa Kumar T. Validation of Potential Fishing Zone (PFZ) forecasts from Andaman and Nicobar Islands. Fishery Technology. 2013; 50 (2013) : 208 - 212.

18. Hyrenbach KD, Veit RR, Weimerskirch H, Hunt GL. "Seabird Associations with Mesoscale Eddies: The Subtropical Indian Ocean." Marine Ecology Progress Series. 2006; 324: 271-279.

19. John ME, Bhargava AK, Varghese S, Gulati DK, Ashok SK, Dwivedi SK. "Fishery Resources of the Indian EEZ around Andaman and Nicobar Islands" Bulletin of Fishery Survey of India. 2005; : 16-38.

20. Leela Edwin, Hridayanathan C. Ring Seines of South Kerala Coast Fishery Technology. 1996; 33(1) pp : 1 - 5.

21. Mcgillicuddy DJ, Robinson AR, Siegel DA, Jannasch HW, Johnson R, Dickey TD, Mcneil J, Michaels AF, Knap $\mathrm{AH}$. "Influence of Mesoscale Eddies on New Production in the Sargasso Sea." Nature. 1998; 394: 263-266. doi:10.1038/28367.

22. Mizobata K, Saitoh SI, Shiomoto A, Miyamura T, Shiga N, Imai K, Toratani M, Kajiwara Y, Sasaoka K. "Bering Sea Cyclonic and Anticyclonic Eddies Observed during Summer 2000 and 2001." Progress in Oceanography. 2002; 55: 65-75. doi:10.1016/S0079-6611(02)00070-8.

23. Mohammed Tahir. Present status and future scope of fisheries in the Andaman group of islands. Journal of the Indian Fisheries Association. 1988; 18: 189-195.

24. Mustafa Am, Dwivedi SN. Abidi SAH. A view towards a Blue Revolution in Andaman and Nicobar Islands SeaPresent status and prospects. Advances in Aquatic Biology and Fisheries. 1987; Pp. 207-223.

25. Nayak SR, Solanki HU, Dwivedi RM. Utilization of IRS P4 ocean colour data for potential fishing zone-A cost benefit analysis. Ind. J. Mar. Sci. 2003; 32(3): 244-248.

26. Nithyanandan R. "Development of Fisheries in Andaman and Nicobar Islands: A case of the Pontential Going Abegging". In S.Dam Doy et.al. (Eds), Proceeding of Brainstarming session on Development of Island Fsheries, Central Agricultural Research Institute, Port Blair. 2009; pp. 1-3.

27. Olson DB, Backus RH. "The Concentrating of Organisms at Fronts: A Cold-Water Fish and a Warm-Core Gulf Stream Ring." Journal of Marine Research. 1985; 43: 113-137. doi:10.1357/002224085788437325.

28. Peter JP, Nelson GJM, Thosaporn W. Clupeoid fishes of the world (suborder Clupeoidei). Rome: United Nations Development Programme. 1988; p 106.

29. Pillai, Abdussamad. "Development of Tuna Fisheries in Andaman and Nicobar Islands". In S.Dam Doy et.al. (Eds), Proceeding of Brainstarming session on Development of Island Fsheries, Central Agricultural Research Institute, Port Blair. 2009; p. 23-34.

30. Rosário Fernandes Jorge Laissane. Artisanal purse seine design improvements suggested for Mozambique fisheries. Fisheries training programme conducted by National Institute for Development of Small-Scale Fisheries, published by United nations university. 2011; p.27

31. Sabarros PS, Ménard F, Lévénez JJ, Kai ET, Ternon JF. "Mesoscale Eddies Influence Distribution and Aggregation Patterns of Micronekton in the Mozambique Channel." Marine Ecology Progress Series. 2009; 395: 101-107.

32. Seki, MP, Polovina JJ, Brainard RR, Bidigare RR, Leonard CL, Foley DG. "Observations of Biological Enhancement at Cyclonic Eddies Tracked with GOES Thermal Imagery in Hawaiian Waters." Geophysical Research Letters. 
2001; 28: 1583-1586.

33. Siar SV, Venkatesan V, Krishnamurthy BN, Sciortino JA. Experiences and lessons from the cleaner fishing harbours initiative in India.2011.

34. Solanki HU, Dwivedi RM, Nayak SR. Synergistic analysis of Sea WiFS chlorophyll concentration and NOAAAVHRR SST features for exploring marine living resources. Int. J. Rem. Sen. 2001; 22: 3877-3882.

35. Solanki HU, Dwivedi RM., Nayak SR, Gulati DK. John ME, Somavanshi VS. Potential Fishing Zone (PFZs) forecast using satellite data derived biological and physical processes. J. Ind. Soci. Rem. Sen. 2003; 31(2): 67-69.

36. Solanki HU, Pradhan Y, Dwivedi RM, Nayak SR, Gulati DK. Somvanshi VS. Application of Quick SCAT Sea Winds data to improve remotely sensed Potential Fishing Zones (PFZs) forecast methodology: Preliminary validation results. Ind. J. Mar. Sci. 2005; 34 (4): 441-448.

37. Solanki HU, Raman M, Kumari B, Dwivedi RM, Narain A. Seasonal trends in the fishery resources off Gujarat: salient observations using NOAA-AVHRR. Ind. J. Mar. Sci. 1998; 27: 438-44

38. Sudarsan D, John ME, Somvanshi VS. Marine fishery resource potential in the Indian exclusive economic zone - an update, Bull. Fish. Surv. India.1990; 20 (1): (1990) 20 -27.

39. Thomas J, Venus S, Kurup BM. Length- weight relationship of some deep-sea fish inhabiting continental slope beyond 250m depth along West coast of India. Naga. World Fish Center Quarterly. 2003; 26: 17-21.

40. Yoder JA, Atkinson LP, Lee TN, Kim HH, Mcclain CR. "Role of Gulf Stream Frontal Eddies in Forming Phytoplankton Patches on the Outer Southeastern Shelf." Limnology and Oceanography. 1981; 26: 1103-1110. 